ARTICLE

Received 9 Sep 2016 | Accepted 12 Dec 2016 | Published 31 Jan $2017 \quad$ DOl: 10.1038/ncomms14249 OPEN

\title{
Pancancer modelling predicts the context-specific impact of somatic mutations on transcriptional programs
}

Hatice U. Osmanbeyoglu ${ }^{1}$, Eneda Toska ${ }^{2}$, Carmen Chan ${ }^{2}$, José Baselga ${ }^{2} \&$ Christina S. Leslie ${ }^{1}$

\begin{abstract}
Pancancer studies have identified many genes that are frequently somatically altered across multiple tumour types, suggesting that pathway-targeted therapies can be deployed across diverse cancers. However, the same 'actionable mutation' impacts distinct context-specific gene regulatory programs and signalling networks-and interacts with different genetic backgrounds of co-occurring alterations-in different cancers. Here we apply a computational strategy for integrating parallel (phospho)proteomic and mRNA sequencing data across 12 TCGA tumour data sets to interpret the context-specific impact of somatic alterations in terms of functional signatures such as (phospho)protein and transcription factor (TF) activities. Our analysis predicts distinct dysregulated transcriptional regulators downstream of somatic alterations in different cancers, and we validate the context-specific differential activity of TFs associated to mutant PIK3CA in isogenic cancer cell line models. These results have implications for the pancancer use of targeted drugs and potentially for the design of combination therapies.
\end{abstract}

\footnotetext{
${ }^{1}$ Computational and Systems Biology Program, Memorial Sloan Kettering Cancer Center, 1275 York Avenue, Box No. 460, New York, New York 10065, USA ${ }^{2}$ Human Oncogenesis and Pathogenesis Program, Memorial Sloan Kettering Cancer Center, New York, New York 10065, USA. Correspondence and requests for materials should be addressed to C.S.L. (email: cleslie@cbio.mskcc.org).
} 
C ancer cells evolve to acquire hallmark capabilities to sustain chronic proliferation, evade growth suppressors and avoid cell death ${ }^{1}$ largely through the accumulation of somatic alterations that disrupt key signalling pathways. Large-scale cancer genomics projects such as The Cancer Genome Atlas (TCGA) have generated a comprehensive catalogue of somatic mutations and copy number aberrations across many tumour types. These alterations have been mapped to known pathways with the hope of deploying pathway-targeted therapeutics-drugs targeting mutant oncoproteins or highly overexpressed wild-type (WT) receptors or signal-transduction proteins-for personalized medicine ${ }^{2-11}$. However, while the same 'actionable mutation' may occur in multiple cancers, it interacts with context-specific regulatory and signalling networks as well as the genetic background of other somatic alterations, suggesting that its impact-and the effectiveness of the targeted therapy-may strongly depend both on the cancer type and additional molecular features of the individual tumour. Moreover, the role of many frequent somatic alterations remains obscure, and it is unclear whether and how they interact with targetable pathways. In fact, computational studies of drug sensitivity across cancer cell lines have found that gene expression features are more informative than mutations for predicting response to targeted therapies ${ }^{12}$. Meanwhile, early 'basket' clinical trials that enroll patients for targeted therapies based on mutation status alone-regardless of cancer type-have demonstrated efficacy only in a subset of cancers 13,14 . These findings point to the need for better integrative computational methods that leverage additional molecular readouts to model the context-specific impact of somatic alterations on gene expression programs.

To this end, we applied a computational strategy for exploiting parallel (phospho)proteomic and mRNA sequencing data for large tumour sets by linking the dysregulation of upstream signalling pathways with altered transcriptional response through the transcriptional circuitry ${ }^{15,16}$. We then developed a statistical framework to interpret the impact of mutations and copy number events in terms of altered (phospho)protein and transcription factor (TF) activity. We used this strategy to train (phospho)protein-TF interaction models across 12 human cancers in TCGA. First, we identified shared and cancer-specific roles of $\mathrm{TF} /$ signalling regulators across cancer types. In bladder urothelial carcinoma, renal cell clear carcinoma and uterus endometrial carcinoma, many of the identified TF regulators were significantly associated with survival outcome. By stratifying tumours by inferred TF activities rather than gene expression patterns, we identified known and previously unlinked TFs that are differentially active in $\operatorname{HPV}(+)$ versus $\operatorname{HPV}(-)$ head and neck squamous cancer, and we uncovered a subtype of endometrioid uterine cancer harbouring mutant $\beta$-catenin with altered TF activities.

We next performed a systematic regularized regression analysis to associate frequent somatic aberrations with changes in inferred $\mathrm{TF}$ and (phospho)protein activities in each cancer type. This analysis identified key regulators associated with the major mutations in renal clear-cell carcinoma. More generally, we observed that specific molecular aberrations have cancerspecific functional consequences. In particular, we associated PIK3CA activating mutations with altered activities of distinct sets of TFs in different cancers. Notably, in isogenic cell line models of breast cancer and head and neck cancer, we validated the altered activity of several TFs in the presence of mutant PIK3CA by measuring promoter occupancy and expression of target genes, confirming the context-specific predictions of our model. These proof-of-principle results suggest a computational strategy for personalized deployment of targeted therapeutics-and potentially for the development of contextspecific combination therapies-in a pancancer setting.

\section{Results}

Pancancer analysis models dysregulated TFs and signalling. We used a computational strategy for exploiting parallel (phospho)proteomic and transcriptomic data to learn a model that links alterations in signalling (from RPPA data) with downstream changes transcriptional response (measured by mRNA data) via predicted TF binding sites ${ }^{15}$ (Fig. 1a-c). We used a regularized bilinear regression algorithm called affinity regression $(\mathrm{AR})^{16}$ to learn an interaction matrix between upstream signal-transduction proteins and downstream TFs that predicts target gene expression (Fig. 1a, bottom). More intuitively, the model learns weighted edges between signalling proteins and TFs to describe the flow of information from change in (phospho)protein level to altered activity of TF to transcriptional changes in target genes (Fig. 1a, top). In a pancancer context, an AR model is trained independently for each cancer type and explains the variation in gene expression across tumours in terms of (phospho)protein variation and presence of TF binding sites (see Methods section).

We can further use the trained AR interaction matrix for each cancer type to obtain different views of each tumour data set via mappings (Fig. 1b): given a tumour sample's (phospho)protein expression levels, we can multiply through the model to infer sample-specific TF activities; conversely, given the gene expression profile, we can multiply through the motif hit matrix and the model to infer '(phospho)protein activities' that are more informative than the original noisy RPPA data (Fig. 1b, bottom). Intuitively, information flows down from observed RPPA levels through the learned interaction matrix to infer TF activities, and observed mRNA expression levels propagate up through the TF-target edges and interaction network to infer (phospho)protein activities (Fig. 1b, top).

Importantly, by associating the presence of somatic alterations with altered regulator activities, we can gain mechanistic insight into the role of specific mutations or copy number events (Fig. 1c). We perform the association analysis by using regularized regression to predict each inferred $\mathrm{TF}$ activity (resp. (phospho)protein activity) individually from the full set of frequent mutation and copy number features (Fig. 1c, bottom; see Methods section). We then evaluate the significance of the effect size (coefficient) for each alteration in the regression model by a permutation approach (see Methods section). After false discovery rate (FDR) correction across TFs/(phospho)proteins, we can identify a significant set of regulators whose altered activities are associated with each mutation/copy number event while controlling for the genetic background of other alterations.

We trained AR models on tumours from 12 different TCGA cancer studies using samples for which mRNA, RPPA, somatic mutation and copy number variation data were available: bladder urothelial carcinoma (BLCA, $n=115)$, breast cancer (BRCA, $n=368$ ), colorectal adenocarcinoma (COADREAD, $n=150)$, glioblastoma multiforme (GBM, $n=58)$, head and neck squamous carcinoma (HNSC, $n=194$ ), kidney renal cellclear carcinoma (KIRC, $n=376$ ), lung adenocarcinoma (LUAD, $n=216$ ), lung squamous cell carcinoma (LUSC, $n=106$ ), ovarian carcinoma (OV, $n=164)$, prostate cancer (PRAD, $n=159)$, uterine corpus endometrial carcinoma (UCEC, $n=183$ ), and uterine carcinosarcoma (UCS, $n=47$ ).

For statistical evaluation, we computed the mean Spearman correlation between predicted and measured gene expression profiles on held-out samples using 10-fold cross-validation for each cancer model. We obtained significantly better performance 
a
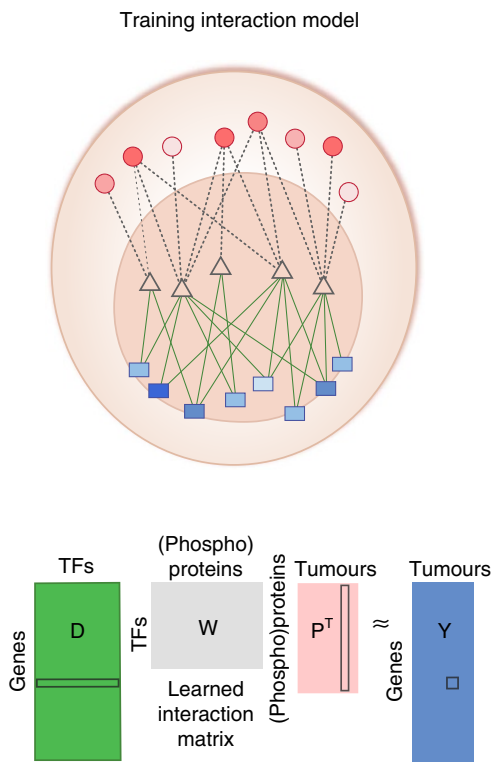

b

Inferring TF and (phospho)protein activities

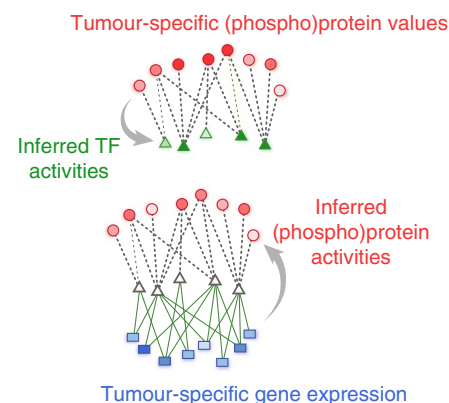

Tumour-specific gene expression

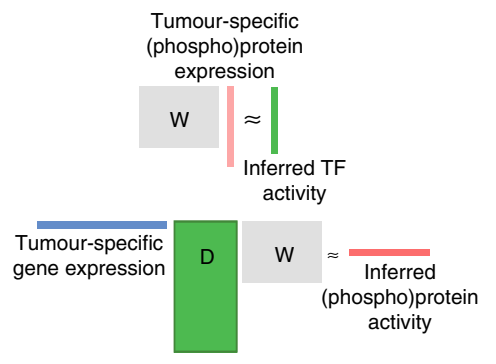

C

Modelling impact of mutations on TF and (phospho)protein activities

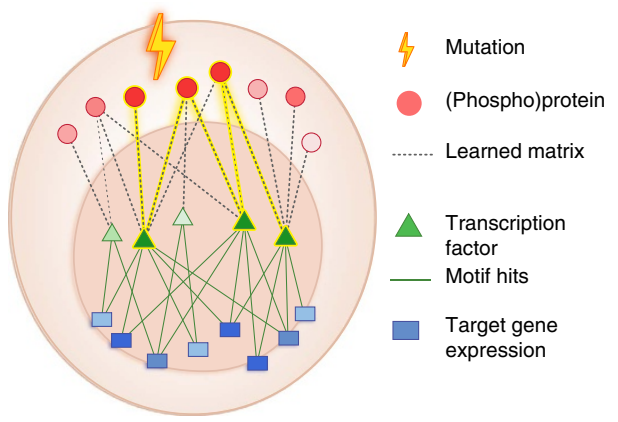

Somatic

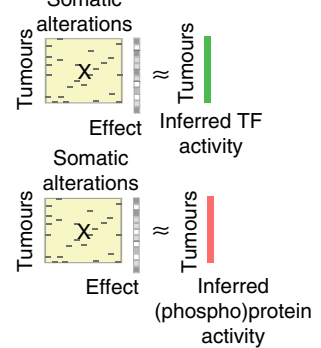

Figure 1 | Integrative computational model links signalling to downstream transcriptional programs. (a) Formally, an interaction matrix $\mathbf{W}$ between TFs and upstream signalling proteins is trained using a bilinear regression algorithm, called affinity regression, on RPPA and mRNA expression (RNA-seq) data from a set of tumours, together with TF binding motif information from gene promoters. The model learns to predict target gene expression from tumour-specific (phospho)protein expression levels and gene-specific TF binding sites. The model can be viewed as learning weighted edges (shown as dashed lines) between upstream signalling proteins (shown as red circles) and transcription factors (TFs, shown as triangles) to capture the flow of information from signalling pathways to TFs to target genes and to predict target gene expression changes (TF to target genes shown in green). Formally, the learned weighted edges between (phospho)proteins and TFs are represented by an interaction matrix. (b) (Phospho)protein-TF interaction models for each cancer are trained independently. The model can be used to infer sample-specific TF activities from measured RPPA profiles or to infer sample-specific (phospho)protein activities from measured mRNA expression values by use of matrix mappings. (c) To model the impact of somatic aberrations on transcriptional response and signalling events, we use regularized regression to predict inferred TF activities (resp. inferred (phospho)protein activities) from somatic alterations. The significance of the effect size (regression coefficient) for each somatic alteration on TF/(phospho)protein activity is estimated by a permutation approach. The eventual goal of the modelling is to understand the cancer-specific downstream effects of targeted therapies and to potentially discover secondary targets for combination drug strategies.

than a nearest-neighbour approach based on Euclidean distance in the RPPA space $(P<0.00025$, one-sided Wilcoxon's signed-rank test; Supplementary Table 1). Similarly, AR models with true motif and RPPA data outperformed models where motif hits for each gene and RPPA profiles for each tumour were randomized $(P<0.00025$, one-sided Wilcoxon's signed-rank test). When only the motif hits were randomized, the performance improvement of the true model was modest but still significant $(P<0.00074$, one-sided Wilcoxon's signed-rank test), suggesting that the motif data, while noisy, contributes to predictive performance. AR obtained similar performance advantages when assessed using a single held-out test set or when evaluating Pearson correlation or root mean-squared error (Supplementary Figs 1-5).

Pancancer AR identifies signatures of survival. To assess the statistical significance of AR-inferred regulator activities, we developed an empirical null model based on training AR models on randomly permuted gene expression profiles for each tumour type (see Methods section). Then, we asked whether the value of individual $\mathrm{TF} /$ (phospho)protein activities for each sample were significantly low or high relative to the corresponding distribution over permuted data. We corrected for FDR across TFs/(phospho)proteins (see Methods section) and identified significant shared and cancer-specific $\mathrm{TF} /($ phospho)protein regulators (Fig. 2a and Supplementary Data 1).

Figure $2 \mathrm{a}$ shows the fraction of samples per cancer type where each TF was identified as a significant regulator; for clarity, only the union of top 10 most prevalent significant TFs per cancer are shown. Certain TFs display a large variation in inferred activity in specific cancer types, suggesting a key role in regulating target gene expression in these cases, while having more modest variation in other cancers. Figure $2 b$ shows the inferred activity distribution of three TFs identified from our analysis: FOXO1 (Forkhead box O), NFE2L2 and ELK1. FOXO1, a key regulator of cell-cycle progression and apoptosis, was identified as a significant regulator for more than $10 \%$ of tumours in BLCA, BRCA and UCEC; its activity showed high variation among tumours for these particular cancers (Fig. 2b, top panel).

A number of TFs were significantly altered in two or more tumour types, including ZEB1, JUN, ELK1, FOXM1, while others were limited to a single type, such as FOXD1 in HNSC and FOXL1 in KIRC. We identified TFs that are known cancer drivers such as STAT5 (endometrioid carcinoma ${ }^{17}$ ), $\mathrm{AHR}^{18}, \mathrm{HMGA}^{19}$ (KIRC), PBX1 $\left(\mathrm{OV}^{20}\right.$, prostate cancer $\left.{ }^{21}, \mathrm{BRCA}^{22,23}\right)$ and NFE2L2 (squamous cell lung cancer). Other predicted TF-cancer relationships are unknown and may provide new mechanistic insights. 

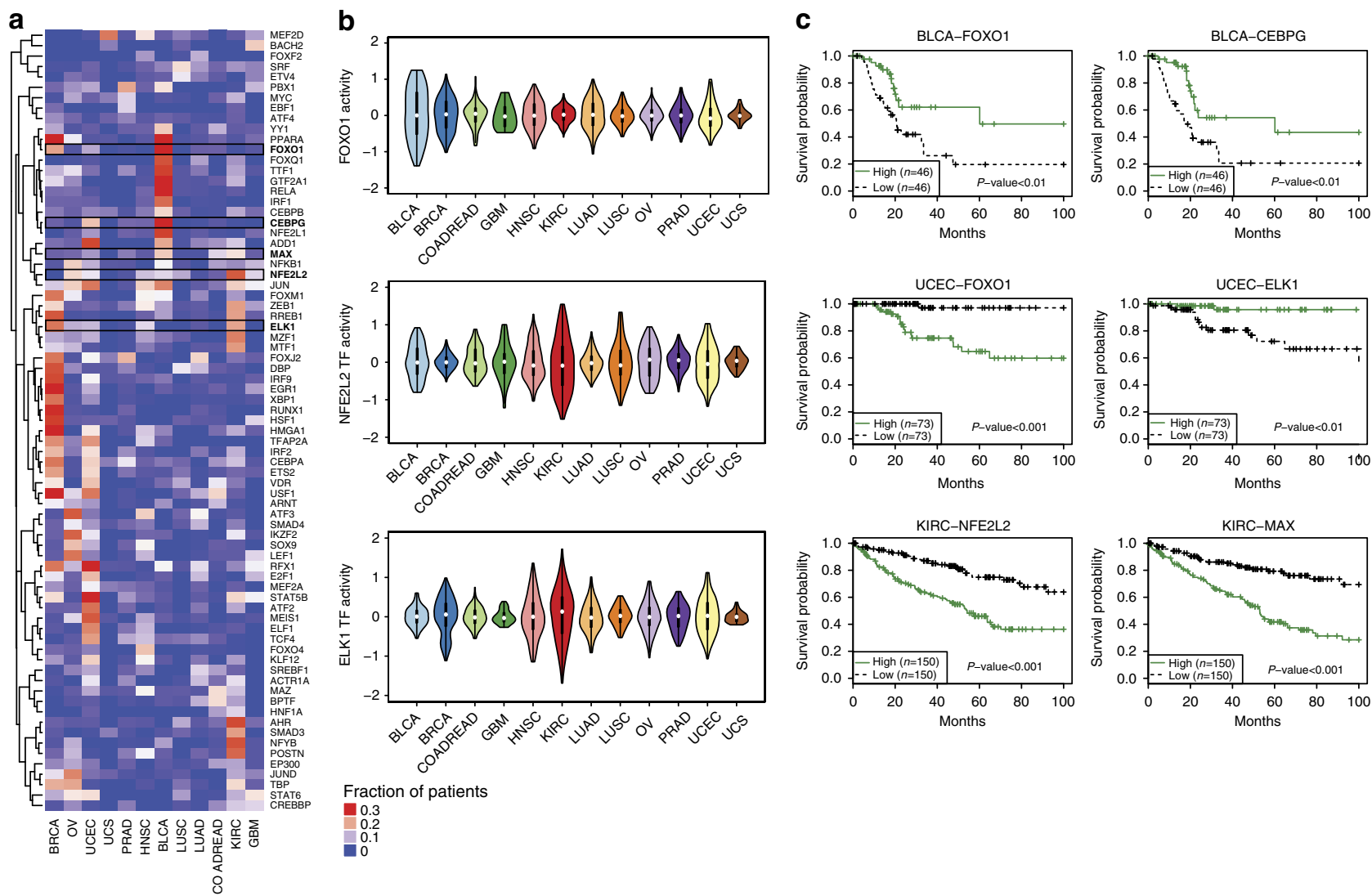

Figure 2 | Activities of significant regulatory TFs correlate with patient survival. (a) Significant TF regulators for each cancer type were identified using an empirical null model based on training affinity regression models randomized gene expression data (see Methods section). A FDR of 10\% was used for studies with $>300$ samples (BRCA, KIRC), 15\% FDR for mid-size studies (BLCA, COADREAD, HNSC, LUAD, LUSC, UCEC, OV, PRAD), 25\% FDR for studies with $<50$ samples (GBM, UCS). The heat map shows the fraction of samples where each TF is identified as a significant regulator within each cancer. For clarity, the union of top 10 most prevalent significant TFs in each cancer-specific model is shown. (b) Violin plots indicate the distribution of inferred FOXO1, NFE2L2 and ELK1 TF activities across cancer types. For example, FOXO1 TF activity is highly variable across tumours in BLCA, BRCA and UCEC. (c) Inferred TF activity predicts survival in patients with BLCA, UCEC and KIRC cancers. Kaplan-Meier survival curves for TCGA BLCA samples, stratified by TF activity of FOXO1 (top left), CEBPG (top right); TCGA UCEC samples stratified by TF activity of FOXO1 (middle left), ELK1 (middle right); TCGA KIRC samples stratified by TF activity of NFE2L2 (bottom left), MAX (bottom right).

To investigate the clinical relevance of these findings, we examined whether the inferred activity of significant TFs was linked to patient survival. We fit Cox proportional hazards regression models for each TF activity using clinical stage (or histological subtype for UCEC) as an additional covariate. Indeed, many identified TF regulators had highly significant associations with survival outcome in BLCA, KIRC and UCEC (Fig. 2c and Supplementary Tables 2-4). For instance, FOXO1 was associated with survival in BLCA and UCEC, and its inferred activity separated patients into high- and low-risk groups. Previous immunohistochemical analyses of FOXO1 in bladder cancer showed that increased mRNA expression is associated with reduced disease progression $^{24}$, consistent with our result. Inferred NFE2L2 and MAX activity were associated with patient survival in KIRC, as was ELK1 activity in the UCEC study. Importantly, Cox models built from inferred TF activities achieved more significant patient stratification than models built from the gene expression of significant TFs (BLCA: $P<10^{-4}$; UCEC: $P<10^{-10}$, one-sided paired Wilcoxon's signed-rank test) (see Methods section and Supplementary Tables 2-4). We further confirmed that most of our UCEC survival results generalized to two other independent cohorts, MDACC (MD Anderson Cancer Center) and Bergen ${ }^{25}$ (see Methods section and Supplementary Table 5), supporting the use of inferred TF activity for patient stratification.

TF activities distinguish HPV $(+)$ and HPV $(-)$ HNSC patients. Next, we asked whether our method could identify known and novel TFs that are differentially active in cancer subtypes. Figure 3 a shows the clustering of tumours by inferred TF activities, together with inferred (phospho)protein activities for the same tumour ordering, as derived from the HNSC model (showing $\mathrm{TF} /$ (phospho)protein activities with largest standard deviation across samples). Notably, patterns of TF activities across tumours generally correlated with (phospho)protein activities.

Head and neck squamous cancer is frequently associated with human papillomavirus (HPV) infection and mutations in TP53. AR analysis suggests that the molecular pathogenesis of $\operatorname{HPV}(+)$ head and neck cancer is distinct from $\operatorname{HPV}(-)$ tumours. Inferred TF activities of 33 TFs were significantly associated with HPV status ( $t$-test, FDR-corrected $P<0.01$, Fig. $3 b, c)$; by contrast, the gene expression values of only two TFs were associated with HPV status (Supplementary Table 6). Altered TF activities were involved in cell-cycle, apoptosis, oxidative stress, WNT signalling and transforming growth 
a
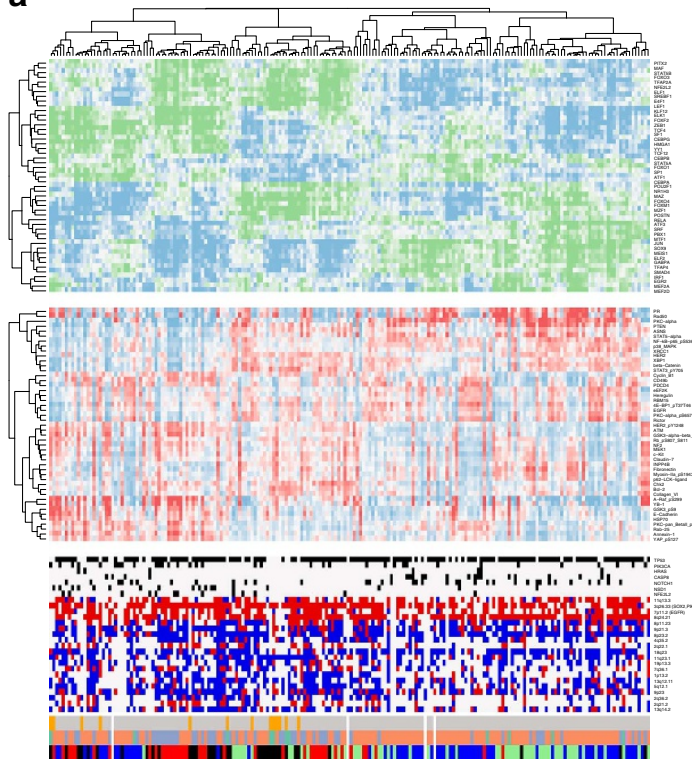

b

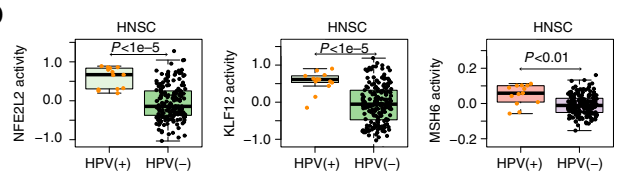

d

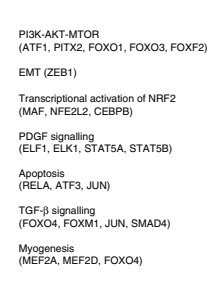

DNA damage response

(RAD50, XACC1)
RTK (ERBB2, EGFR)

TSC-mTOR (RICTOR, EIFAEBPP)

PI3K-AKT (GSK3A)

Ras.MAPK
(ARAF, MAP2K1, MAPK14)

EMT

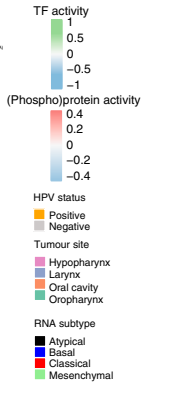

质

e
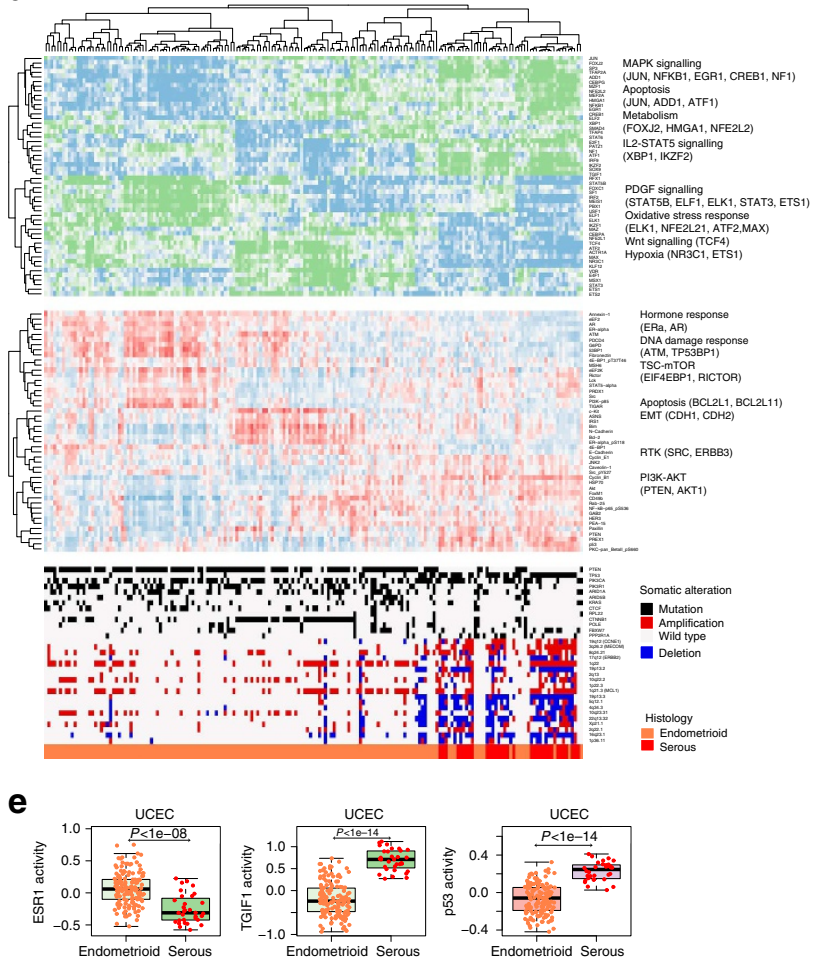

C

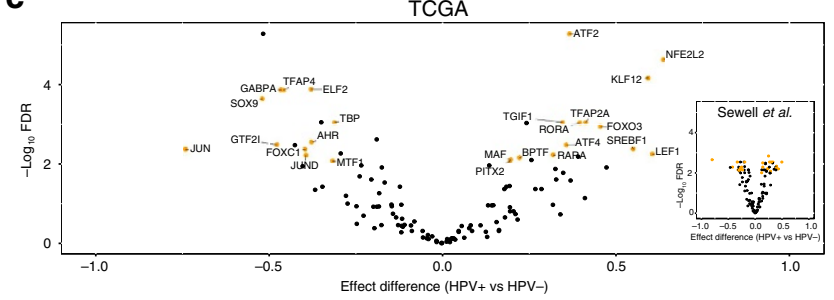

f

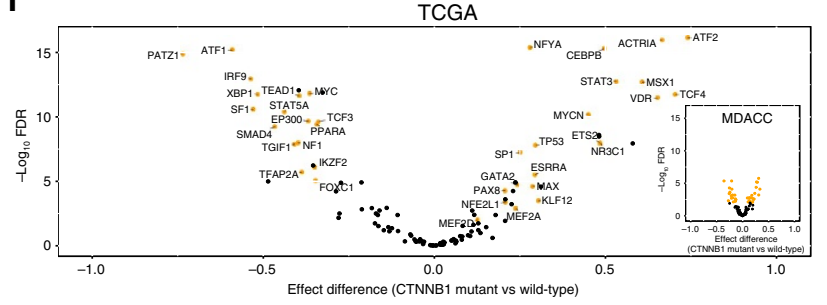

Figure 3 | Pancancer affinity regression modelling identifies regulatory features of tumour subtypes. (a) We trained an affinity regression model on 194 tumours from the TCGA HNSC study. The top heat map shows tumours clustered by the inferred TF activities with highest variance. The middle panel shows top most variable ( 50) inferred (phospho)protein activities for each tumour based on clustering by sample-specific TF activities. The bottom panel shows genomic aberration profiles of each tumour as well as HPV status, tumour site and mRNA expression subtypes derived from the corresponding TCGA HNSC study. (b) Examples of three of the significantly differential inferred TF/protein activities in HPV $(+)$ versus HPV $(-)$ tumours were NFE2L2, KLF12 and MSH6. HPV $(+)$ tumours have significantly higher NFE2L2 TF activity $\left(P<10^{-5}\right.$, Wilcoxon's rank-sum test), higher KLF12 TF activity $\left(P<10^{-5}\right.$, Wilcoxon's-rank sum test) and higher MSH6 protein activity $\left(P<10^{-2}\right.$, Wilcoxon's rank-sum test) than $H P V(-)$ tumours. (c) The mean inferred TF activity difference in HPV $(+)$ and HPV $(-)$ patients is plotted on the $x$ axis, and FDR-adjusted significance from $t$-test is plotted on the $y$ axis (- $\log 10$ scale) for TCGA and Sewell et al. ${ }^{28}$ head and neck cancer cohorts. TFs significantly associated with HPV status (FDR $\left.<0.01\right)$ in both cohorts are coloured in orange. (d) We trained an affinity regression model on 183 tumours from the TCGA endometrial carcinoma (UCEC) study. The top heat map shows a clustering of tumours by inferred TF activities. The middle panel shows inferred (phospho)protein activities of each tumour based on clustering of tumour TF activities. The bottom panel shows genomic aberration profiles of each tumour as well as histological subtypes derived from the corresponding TCGA UCEC study. Patterns of TF activities across tumours often correlated with patterns of (phospho)protein activities. (e) Examples of three of the significantly differential inferred TF/(phospho)protein activities in serous versus endometrioid tumours were ESR1, TGIF1 and p53. Tumours with endometrioid histology have significantly higher ESR1 TF activity $\left(P<10^{-8}\right.$, Wilcoxon rank sum test), lower TGIF1 TF activity $\left(P<10^{-14}\right.$, Wilcoxon rank-sum test), and lower p53 protein activity $\left(P<10^{-14}\right.$, Wilcoxon rank sum test) than serous tumours. (f) The mean inferred TF activity difference in CTNNB1 mutant and CTNNB1 wild-type patients is plotted on the $x$ axis, and false discovery rate (FDR)-adjusted significance from $t$-test is plotted on the $y$ axis ( $-\log 10$ scale) for TCGA and MDACC endometrial cancer cohorts. TFs significantly associated with CTNNB1 status (FDR $<0.01$ ) in both cohorts are coloured in orange. Box edges represent the upper and lower quantile with median value shown as bold line in the middle of the box. Whiskers represent 1.5 times the quantile of the data.

factor- $\beta$ ) signalling and may have roles in the initiation and maintenance of $\operatorname{HPV}(+)$ head and neck cancer ${ }^{26}$. For example, KLF12 and NFE2L2 were significantly associated with $\operatorname{HPV}(+)$ tumours (Fig. 3b). Interestingly, the KLF12 locus is a frequent integration site for the HPV virus ${ }^{27}$ in cervical cancer, and the TCGA HNSC study also identified $K L F 5$, the locus of a related KLF factor, as an HPV integration site $^{5}$. To confirm our results, we used the HNSC TCGA-trained AR model to infer TF activities in an independent set of 42 head and neck cancer RPPA profiles with HPV status ${ }^{28}$. We again identified TF-HPV status associations by $t$-test and found a similar set of TFs (23 out of 33) whose activities significantly differed between $\operatorname{HPV}(+)$ and $\operatorname{HPV}(-)$ tumours; the 33 identified TFs were also enriched among the top-ranked 
TFs in the new cohort $\left(P<10^{-5}\right.$, Mann-Whitney test) (Fig. 3c and Supplementary Table 6).

As described previously ${ }^{29}$, mutant TP53 tends to be mutually exclusive with $\operatorname{HPV}(+)$ status, but inferred TP53 TF activity and inferred $\mathrm{p} 53$ protein activity were not significantly different between $\operatorname{HPV}(+)$ and $\operatorname{HPV}(-)$ patients $(t$-test $P=0.477$ and $P=0.741$, respectively). However, it is known that the viral E6 oncoproteins in $\mathrm{HPV}(+)$ head and neck cancer form a complex with WT p53 and lead to its degradation ${ }^{30}$, pointing to an alternative mechanism for p53 inactivation in $\operatorname{HPV}(+)$ patients.

We performed similar analyses for other TCGA cancer studies and in each cancer type could stratify patients by regulator activity profiles (Supplementary Figs 6-15). For example, the inferred TF activity of CEBPA $^{31}$ was significantly higher in the mesenchymal subtype compared to other subtypes of GBM $\left(P<10^{-5}\right.$, Wilcoxon's rank-sum test used for all tests); ESR1 (estrogen receptor 1 ) activity was higher in luminal BRCA compared to other BRCA subtypes $\left(P<10^{-42}\right)$, consistent with oestrogen receptor serving as a luminal marker ${ }^{32}$; and activity of TTF-1 (thyroid transcription factor-1) thyroid transcription factor-1, a known biomarker of $\mathrm{LUAD}^{33}$, was higher in the squamoid $\left(P<10^{-10}\right)$ and bronchioid subtypes $\left(P<10^{-5}\right)$ compared to the magnoid subtype in LUAD.

TF signature defines mutant CTNNB1 endometrioid subtype. We then asked if we could associate inferred TF activities with mutational signatures as a first step towards developing a more general statistical strategy. Figure $3 \mathrm{~d}$ shows a clustering of tumours by inferred TF activities from the UCEC model, together with inferred (phospho)protein activities and recurrent somatic mutations and copy number events. Serous-like endometrial tumours are hormone receptor negative, mostly copy number high, and harbour mutations in TP53, whereas endometrioid tumours are hormone receptor positive, copy number low, and have a high frequency of PI3K-AKT (phosphatidylinositol 3-kinase-AKT) pathway alterations ${ }^{5,6}$. Consistent with their distinct molecular and genomic features, we found significant differences in inferred regulator activities in serouslike and endometrioid tumours (Supplementary Tables 7 and 8), including increased ESR1 activity in endometrioid tumours $\left(P<10^{-8}\right.$, Wilcoxon's rank-sum test used for all tests $)$ and increased TGIF1 TF activity and inferred p53 protein activity serous-like tumours $\left(P<10^{-14}\right.$ for both tests; Fig. 3e).

Importantly, clustering by $\mathrm{TF}$ activities revealed subclasses of tumours within each histological subtype that sometimes correlated with mutation status. In particular, endometrioid tumours with a CTNNB1 mutation form a distinct cluster based on inferred TF activity profiles that was not observed by clustering TF mRNA expression levels directly (Supplementary Fig. 16). Moreover, clustering based on inferred TF activity was better able to stratify patients by CTNNB1 mutation status $\left(P<10^{-17}\right.$, two-sided $\chi^{2}$ test for all tests) compared to reported TCGA mRNA clusters $(P<0.01)$ and TCGA integrated clusters $\left(P<10^{-6}\right)$ (Supplementary Tables 9 and 10). Significant inferred TF activity differences between CTNNB1 mutant and WT patients (satisfying FDR-corrected $P<0.01, t$-test) associated CTNNB1 mutant status with altered activity of TFs involved in WNT signalling, epithelial-mesenchymal transition and cancer stem cell transition including TCF4 (transcriptional factor 4), NFATC4, JUN, TP53, MAX, MYC, STAT3 and KLF12 (Fig. 3f). We confirmed these results in an independent data set of 203 endometrial RPPA profiles along with mutation and clinical data compiled by MDACC ${ }^{25}$, using the UCEC TCGA-trained AR model to infer TF activities, and replicated many of the TFs associated with mutant CTNBB1 $\left(P<10^{-5}\right.$, Mann-Whitney test; Fig. 3f and Supplementary Table 11). Interestingly, another study performed customized consensus clustering on TCGA UCEC expression data and did identify a cluster enriched with $\beta$-catenin mutations, and GSEA (gene set enrichment analysis) suggested an association with WNT signalling, consistent with our analysis ${ }^{34}$.

Modelling reveals impact of mutations in kidney cancer. Encouraged by our findings for mutant CTNBB1 endometrioid tumours, we developed a systematic statistical approach for modelling the impact of somatic alterations on regulator activity in each tumour type, with the eventual goal of deciphering cancer-specific downstream effects of targeted therapies and potentially discovering secondary targets for combination drug strategies. First, we implemented a regularized regression approach that uses somatic alterations to explain inferred $\mathrm{TF} /$ (phospho)protein activity across tumour samples on a regulator-by-regulator basis. For a complex genotype, the model explains $\mathrm{TF} /($ phospho)protein regulator activity across tumours as the sum of effects of individual somatic alterations (that is, coefficients in the regression model), and the effect size of each alteration is assigned a nominal $P$ value by a permutation approach (see Methods section). We then corrected for multiple hypotheses across regulator models, treating inferred TF activities and inferred (phospho)protein activities as separate groups of tests (see Methods section). Combining these results identified a set of regulators predicted to be significantly dysregulated by each somatic alteration in each TCGA cancer study.

Figure 4a,b shows the regulator activities associated with somatic aberrations in KIRC. Our model identified mutations in VHL (von Hippel-Lindau), PBMR1, BAP1, MTOR, ATM, SETD2, KDM5C and PTEN (phosphatase and tensin homolog), as well as copy number changes in MLH1, DUSP1 and $R A N D B P 17$ as significantly associated with various TF activity changes across tumours. KIRC is characterized by a highfrequency inactivating mutation in the $V H L$ gene found in $\sim 54 \%$ of tumours in TCGA and likely more prevalent ${ }^{35}$. Mutually exclusive mutations in PBRM1, a subunit of the PBAF SWI/SNF chromatin remodelling complex, and in histone deubiquitinase BAP1 define two genetic subtypes of KIRC, while recurrent mutations in the histone methyltransferase SETD2 also occur.

KIRC samples with $P B M R 1$ and $B A P 1$ mutations showed distinct patterns of $\mathrm{TF}$ and protein activities (Fig. 4a), and regression analysis associated different regulators with these mutations (Fig. 4b). PBMR1 mutant tumours are associated with increased activity of $\mathrm{TFs} /($ phospho)proteins that have roles in interleukin signalling and MYC, while regulators with increased activity in BAP1 mutant tumours are involved in DNA damage response, apoptosis, insulin signalling and mTOR signalling.

Notably, NFE2L2 TF activity was significantly higher in BAP1 mutant tumours than PBMR1 mutant tumours. Dysregulation of the KEAP1-NFE2L2 pathway occurs through both genetic and epigenetic mechanisms and induces prosurvival genes promoting proliferation and chemoresistance ${ }^{36}$. Mutations in KEAP1, NFE2L2 (Nrf2), CUL3 or RBX1 are the most common mechanisms that impair KEAP1-mediated degradation of NFE2L2 and thereby activate the transcriptional effects of NFE2L2. Somatic aberrations in these genes have been described in LUSC, LUAD and HNSC, and indeed we confirmed this activating effect (Fig. 4c). Inferred TF activity of NFE2L2 was increased in mutant versus WT KEAP1 or NFE2L2 lung cancers; these differences are not observed at the gene expression level (Supplementary Fig. 17). 


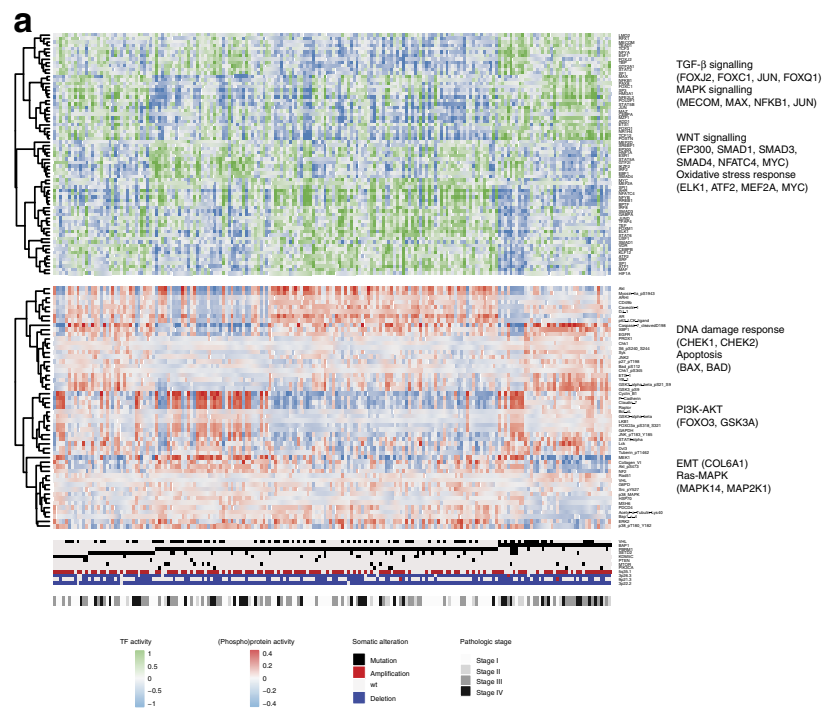

b
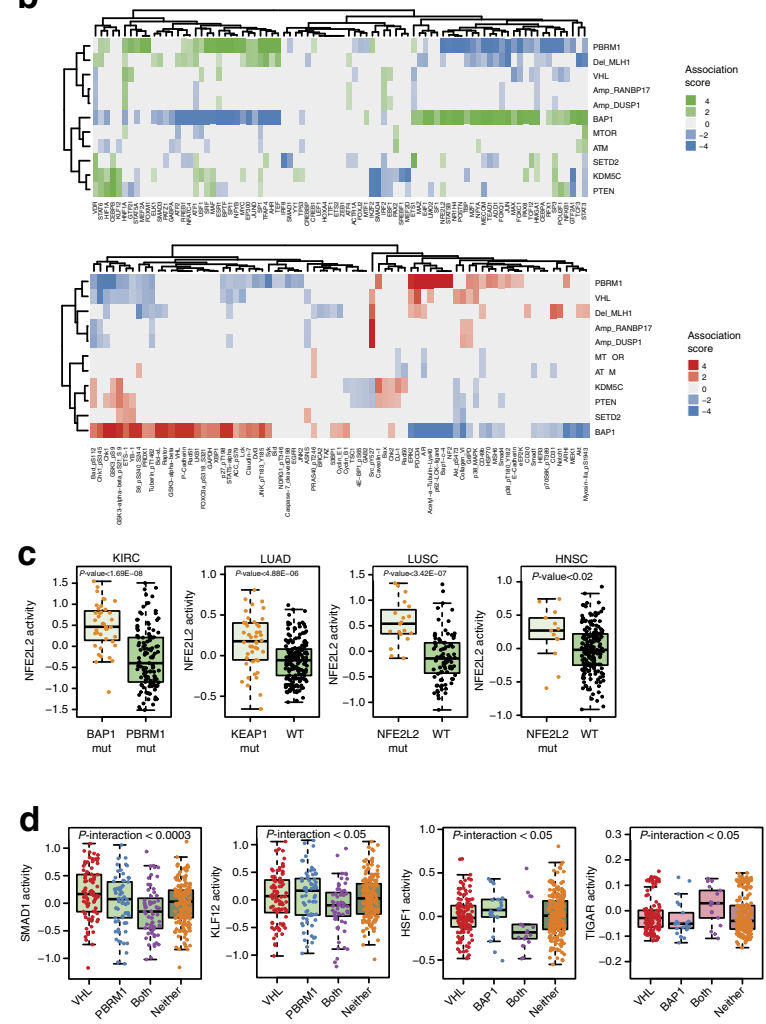

Figure 4 | Genomic aberrations are associated with dysregulated TF and (phospho)protein activity in the TCGA KIRC study. (a) We trained an affinity regression model on 376 tumours from the TCGA KIRC study. The top panel shows inferred TF activity for each tumour associated with BAP1, PBMR1, SETD2 and KDM5C mutations, ordered according to the mutation profile. The middle panel shows the corresponding (phospho)protein activity for each tumour associated with BAP1, PBMR1, SETD2 and KDM5C mutations. The bottom panel shows genomic aberration profiles of each tumour as well as the pathological stage as derived from the corresponding TCGA KIRC study. (b) Impact of genomic aberrations on individual TF/(phospho)protein activities in TCGA KIRC, based on a regularized regression analysis. A permutation test approach was used to assign significance to ridge regression coefficients (see Methods section). The heat map shows - $\log 10$ FDR-adjusted $P$ values derived by permutation test, multiplied by the sign of the coefficient. The top panel shows inferred TF activity associations. The bottom panel shows inferred (phospho)protein activity coefficients. (c) Inferred NFE2L2 activity in TCGA KIRC, LUAD, LUSC and HNSC studies and impact of mutations using integrative modelling. In the KIRC study, tumours with mutant BAP1 have significantly higher NFE2L2 activity than mutant PBRM1 tumours $\left(P<1.68 \times 10^{-8}\right.$, Wilcoxon's rank-sum test). Tumours with mutant KEAP1/NFE2L2 have also significantly higher inferred TF activity of NFE2L2 (a substrate targeted by KEAP1) than WT tumours in the LUAD study $\left(P<4.88 \times 10^{-6}\right.$, Wilcoxon's ranksum test), LUSC study $\left(P<3.42 \times 10^{-6}\right.$, Wilcoxon's rank-sum test) and HNSC study $(P<0.02$, Wilcoxon's rank-sum test). This association is not significant using the original measured gene expression values of NFE2L2 (Supplementary Fig. 17). (d) Inferred SMAD1 and KLF12 activity is significantly and synergistically decreased in tumours with both VHL and PBMR1 mutations (SMAD1: interaction, $P<0.0003$; KLF12: interaction, $P<0.05$ ). In tumours with both VHL and BAP1 mutations, HSF1 activity is significantly decreased (interaction $P<0.05$ ) and TIGAR protein activity is significantly increased (interaction $P<0.05$ ). Box edges represent the upper and lower quantile with median value shown as bold line in the middle of the box. Whiskers represent 1.5 times the quantile of the data.

We also assessed synergistic effects by building linear models with interaction terms for each pair of somatic alterations (see Methods section). In samples where VHL was comutated with PBMR1, SMAD1 (interaction $P<0.0003$ ) and KLF12 (interaction $P<0.05$ ) activity were significantly decreased. Meanwhile, when VHL was comutated with BAP1, HSF1 activity was significantly decreased (interaction $P<0.05$ ), while TIGAR protein activity was significantly increased (interaction $P<0.05$ ) (Fig. 4d).

PI3K pathway mutations dysregulate cancer-specific TFs. The PI3K pathway controls proliferation, metabolism, survival and motility and is frequently activated in many cancers, often via mutations in PIK3CA, which encodes the $\alpha$-isoform of the p110 catalytic subunit of PI3K (PI3K $\alpha)$; loss of PTEN, which antagonizes PI3K function; and overexpression of membranebound receptor tyrosine kinase $\mathrm{s}^{37,38}$. As PI3K inhibitors are currently in early-phase or phase III trials for use across multiple cancers $^{14,39}$, we asked whether PI3K pathway alterations dysregulate the same or differing TFs and (phospho)proteins across tumour types. Figure 5a and Supplementary Fig. 18 show the regulators associated with somatic aberrations in PTEN and PIK3CA by our analysis in BRCA, HNSC, UCEC, KIRC, LUAD and PRAD tumours (see Methods section).

Activating mutations in PIK3CA were present in $\sim 31 \%$ of BRCA tumours and 20\% of HNSC tumours ${ }^{5}$. Mutations often occur in one of three hotspot locations (E545K, E542K and H1047) and promote constitutive signalling though the pathway. In UCEC, $\sim 66 \%$ of tumours have PTEN inactivating mutations, $\sim 50 \%$ have PIK3CA activating mutations and $\sim 35 \%$ have a comutation of PTEN and PIK3CA. Figure 5a shows 134 TFs associated with somatic aberrations in PTEN or PIK3CA in BRCA, HNSC or UCEC. Notably, the number of TFs dysregulated by PI3K pathway alterations varied widely across different cancers ( 9 in HNSC, 65 in BRCA and 63 in 
a

a

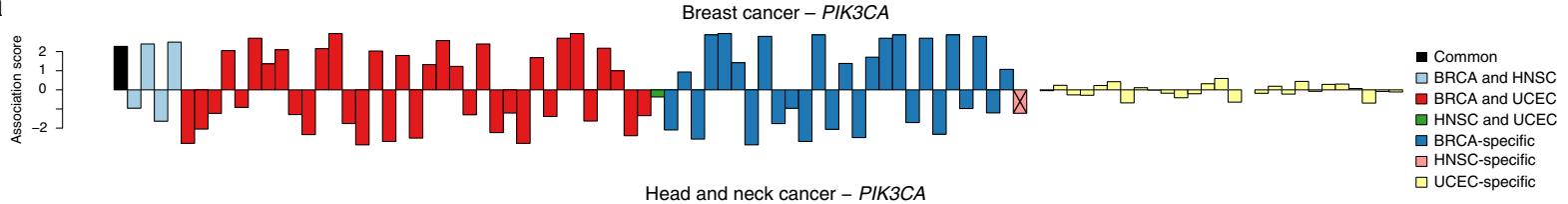

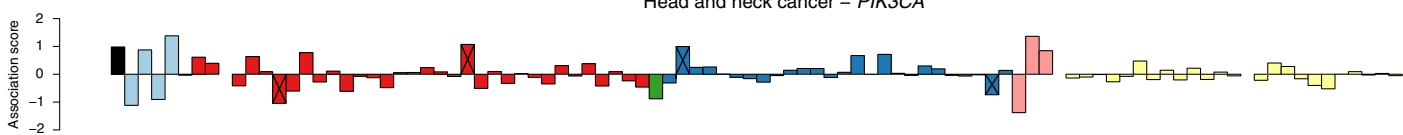

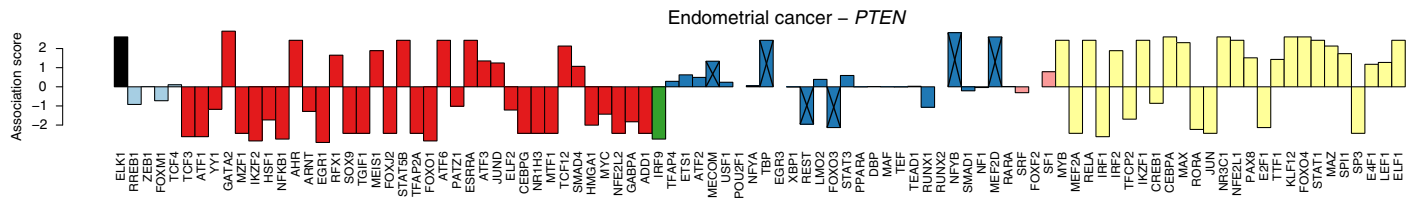

b
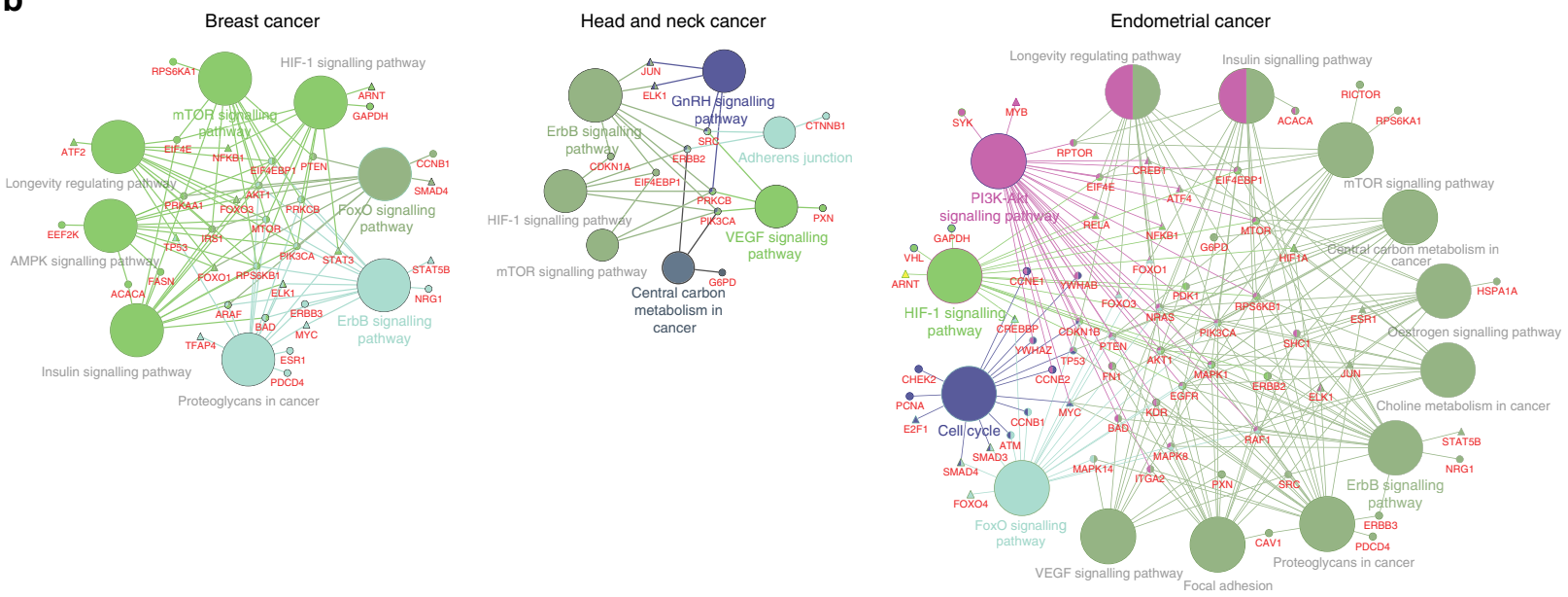

Figure 5 | Somatic aberrations in the PI3K pathway dysregulate cancer-specific TFs. (a) Bar plots show 134 TFs associated with somatic aberrations in PTEN or PIK3CA discovered from regularized regression analysis in breast cancer (BRCA), head and neck squamous carcinoma (HNSC) or UCEC and their association scores. TFs that are significantly associated with the somatic aberration but not identified as significant regulators for explaining gene expression in at least $1 \%$ of samples are indicated with crossed lines. (b) A functionally grouped network of enriched categories was generated for TFs associated with PIK3CA/PTEN mutations using KEGG pathway terms related to cancer as nodes and linked using ClueGO $^{79}$ analysis. Only the most significant terms in the group are labelled. Functionally related groups partially overlap.

UCEC), with striking changes in ErbB/MAPK, mTOR, HIF-1, VEGF and PI3K-Akt pathways. Only ELK1, a TF downstream of the MAPK/ERK pathway, is dysregulated by PIK3CA or PTEN mutations in all three cancers. Four TF associations were shared in BRCA and HNSC: ZEB1 (EMT activator), TCF4 (Wnt signalling), RREB1 (Ras signalling) and FOXM1 (cell proliferation, cell cycle progression, cell differentiation, DNA damage repair, tissue homeostasis, angiogenesis and apoptosis); 35 TFs were common to BRCA and UCEC; and just IRF9 was shared between UCEC and HNSC. There were also associations unique to each cancer type, including USF1 and SMAD1 for BRCA, FOXF2 for HNSC, and NFE2L2 and NR3C1 for UCEC.

Interestingly, many $\mathrm{TF}$ activities were associated with mutant PTEN irrespective of PIK3CA status in endometrial cancer (Supplementary Fig. 19), consistent with a recent preclinical study ${ }^{40}$, while PIK3CA mutations were only significantly associated with a single TF, CREB1. Therefore, PTEN and PIK3CA appear to have distinct consequences for PI3K activation in UCEC.

PI3K pathway inhibition is known to alter STAT5 (ref. 41), FOXO, RUNX2 (ref. 42), ERG1 (ref. 43) and ETS1 (ref. 44) activities, consistent with our results. We also examined protein microarray-based AKT1 kinase assay and SILAC-based phosphoproteomic data from isogenic knock-in breast cell lines harbouring mutations of PIK $3 \mathrm{CA}^{45}$ (see Methods section). Of 11 TFs represented in the phosphoproteomic data and associated with mutant PIK3CA in BRCA, eight of them-ADD1, FOXO3, HMGA1, HSF1, JUND, NF1, POU2F1, STAT3-showed protein abundance change in isogenic cell line systems (see Methods section and Supplementary Table 12). Moreover, of the six TFs represented in the AKT1 kinase assay ${ }^{45}$ and associated with mutant PIK3CA in BRCA, four of them-ETS1, ATF6, SOX9 and TEAD1-were identified as AKT substrates (Supplementary Table 13).

Predicted TFs for mutant PIK3CA validate experimentally. Our analysis associated mutant PIK3CA with ELK1 and TCF4 activity in both breast and head and neck cancer, and with FOXO1 activity in BRCA but not in head and neck cancer. We validated these predictions in isogenic BRCA and head and neck cancer cell lines by measuring promoter occupancy via chromatin immunoprecipitation-quantitative PCR (ChIP-qPCR) and expression change via quantitative reverse transcription-qPCR (RT-qPCR) of canonical target genes of these TFs (Fig. 6 and Methods section). 
a

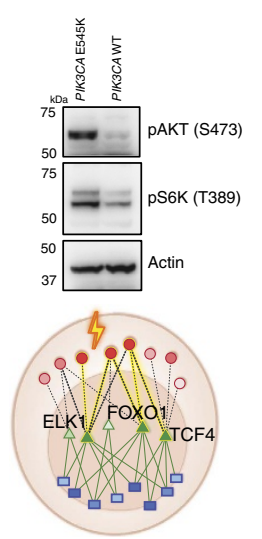

e

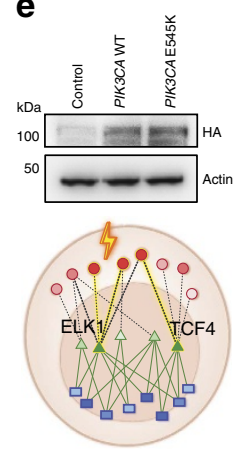

b
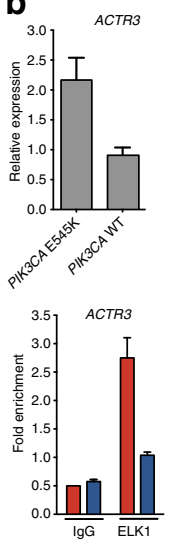

$f$
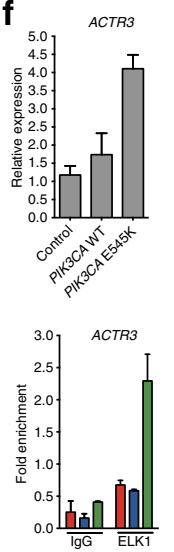
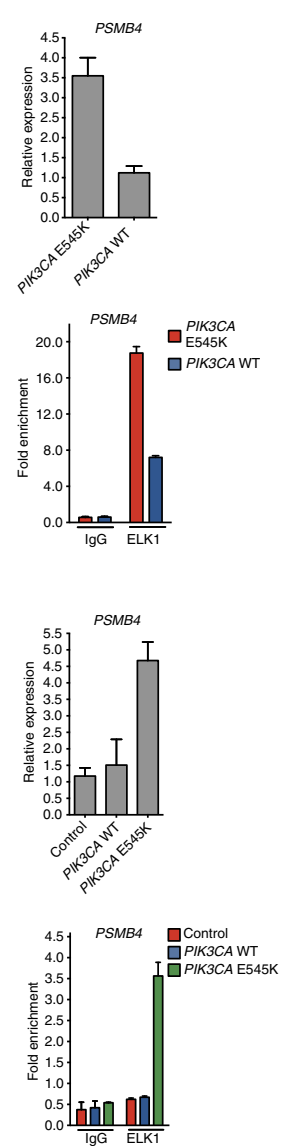

C
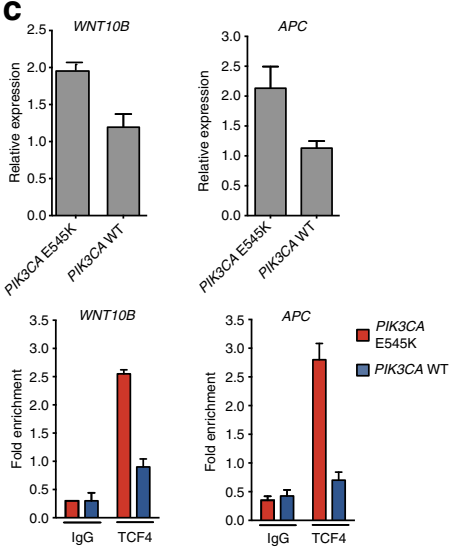

g
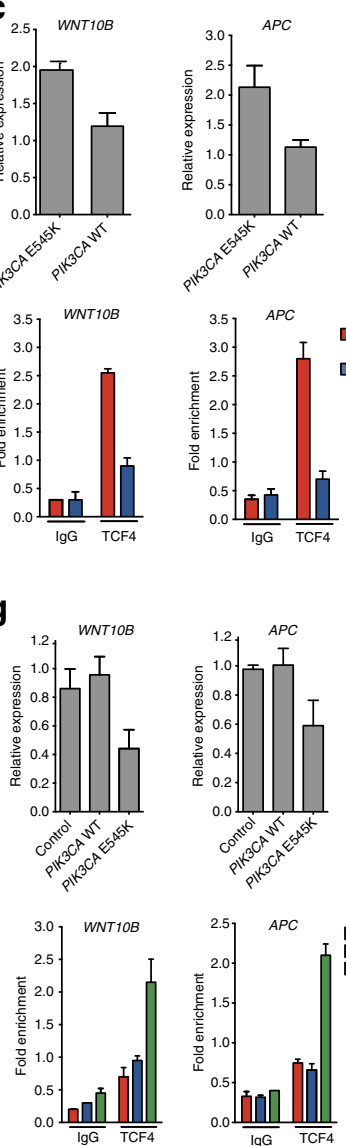

d

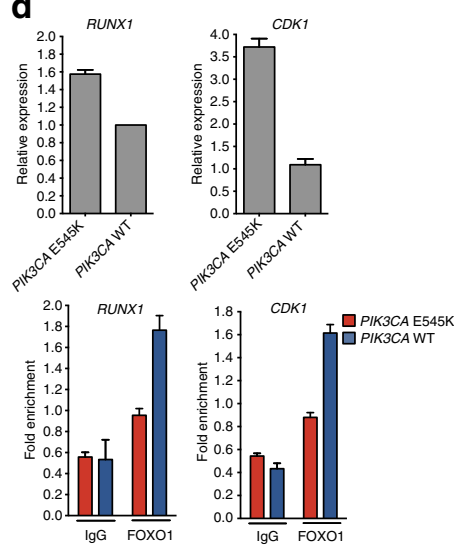

$\mathbf{h}_{1.6}$

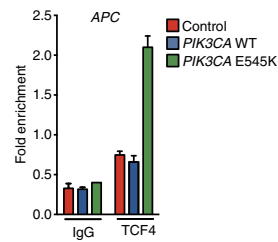

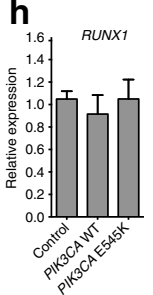
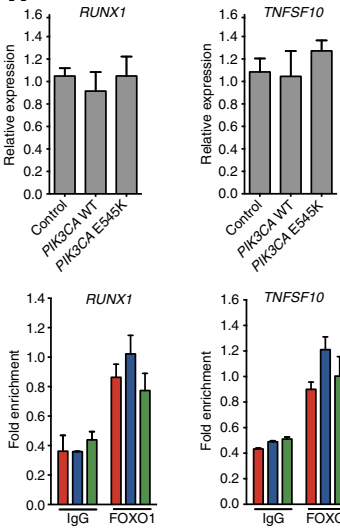

Figure 6 | Predicted transcriptional regulatory impacts of activating PIK3CA mutations validated experimentally. (a) Western blot analysis of pAKT (S473), pS6K (T389) and actin in parental MCF7 cells that carry the PIK3CA E545K mutation and in 'corrected' WT PIK3CA cells. ELK1 activity in PI3K $\alpha$ mutant cells. (b) ACTR3 and PSMB4 mRNA expression in parental PIK3CA mutant and PIK3CA WT cells. ChIP assays with control lgG or ELK1 antibodies for ACTR3 and PSMB4 in parental PIK3CA mutant and PIK3CA WT MCF7 cells. The data are presented as fold-enrichment relative to the actin control gene region. TCF4 activity in PI3K $\alpha$ mutant cells (mean \pm s.d., $\mathrm{n}=3$ independent experiments). (c) WNT10B and APC mRNA expression in parental PIK3CA mutant and PIK3CA WT cells. ChIP assays with control lgG or TCF4 antibodies for WNT10B and APC in parental PIK3CA mutant or PIK3CA WT MCF7 cells. The data are presented as fold-enrichment relative to the actin control gene region (mean \pm s.d., $\mathrm{n}=3$ independent experiments). FOXO1 activity in PI3K $\alpha$ mutant cells. (d) RUNX1 and CDK1 mRNA expression in parental PIK3CA mutant and PIK3CA WT MCF7 cells. Parental and WT MCF7 cells were subjected to ChIP assays with control IgG or FOXO1 antibodies. The data are presented as fold-enrichment relative to the actin control gene region (mean $\pm \mathrm{s}$.d., $\mathrm{n}=3$ independent experiments). (e) Transfected vector control, WT PIK3CA or PIK3CA E545K Cal27 cells were subjected to western blots with haemagglutinin (HA) and actin antibodies after $48 \mathrm{~h}$ of transfection. (f) ACTR3 and PSMB4 mRNA expression in control, PIK3CA WT and PIK3CA E545K cells. ChIP assays with control lgG or ELK1 antibodies in control, WT and PIK3CA E545K cells (mean \pm s.d., $\mathrm{n}=3$ independent experiments). (g) WNT10B and APC mRNA expression in control, PIK3CA WT- and PIK3CA E545K-transfected Cal27 cells. ChIP assays with control lgG or TCF4 antibodies in control-, PIK3CA WT- and PIK3CA E545K-transfected Cal27 cells (bottom panel). (h) RUNX1 and TNSF10A mRNA expression in control, PIK3CA WT- and PIK3CA E545K-transfected Cal27 cells. ChIP assays with control IgG or FOXO1 antibodies in control-, WT- and PIK3CA E545K-transfected Cal27 cells. For other TF targets see Supplementary Fig. 21 and Supplementary Fig. 23.

First, we used the parental MCF7 cell line carrying the PIK3CA E545K mutation and an MCF7 PIK3CA WT cell line in which the mutation was corrected using gene targeting ${ }^{46}$. Western blotting confirmed that WT PIK3CA cells have very low PI3K pathway activation compared to mutant parental cells, with strongly reduced levels of phospho (p)-AKT and p-S6K (Fig. 6a and Supplementary Fig. 20).

Quantitative RT-qPCR analysis of the well-described ELK1 target genes ACTR3, PSMB4 (ref. 47), WNK1, PAPLN, FOXP4 and DDX27 confirmed significant increases in mRNA levels in the parental PIK3CA mutant cells compared to PIK3CA WT cells, with the exception of PAPLN, where we observed a significant decrease (Fig. 6b, top panel; Supplementary Fig. 21a). Moreover, ChIP-qPCR experiments confirmed that ELK1 binding to all five target gene promoters was significantly increased in the PIK3CA mutant MCF7 compared to WT cells, showing that mutant
PIK3CA enhances ELK1 transcriptional activity in BRCA cells (Fig. 6b, bottom panel).

Well-known TCF4 target genes such as WNT10B, APC, $F B X W 11$ and PPP2R5E were differentially regulated by the PIK3CA E545K mutation in MCF7 cells (Fig. 6c, top panel and Supplementary Fig. 21b), and ChIP-qPCR analysis confirmed enhanced binding of TCF4 to their promoters in mutant PIK3CA cells (Fig. 6c, bottom panel and Supplementary Fig. 20b). Similarly, PCR with reverse transcription (RT-qPCR) analysis of FOXO1 target genes RUNX1, CDK1, CAMKK1 and TNFSF10 (ref. 48) mRNA confirmed that their mRNA levels were differentially regulated by the PIK3CA E545K mutation (Fig. 6d, top panel and Supplementary Fig. 21c), and ChIP-qPCR analysis confirmed increased binding of FOXO1 to their promoters in the wild-type PIK3CA MCF7 cells relative to parental PIK3CA E545K cells (Fig. 6d, bottom panel and Supplementary Fig. 21c). 
We also performed validation experiments in the head and neck cancer cell line Cal27, which is WT for the PIK3CA gene. Control, PIK3CA WT or PIK3CA E545K vectors were overexpressed in Cal27, and ChIP-qPCR and RT-qPCR expression experiments were performed to investigate the activity of ELK1, TCF4 and FOXO1. Western blotting confirmed successful expression of these vectors in the Cal27 cell line (Fig. 6e and Supplementary Fig. 22).

Like in the MCF7 BRCA model, RT-qPCR analysis demonstrated an increase in the mRNA levels of four known ELK1 target genes, ACTR3, PSMB4, WNK1 and DDX27, in Cal27 cells transfected with PIK3CA E545K compared to Cal27 cells transfected with WT PIK3CA and control cells (Fig. 6f, top panel and Supplementary Fig. 23a). Increased occupancy of ELK1 at target promoters was confirmed by ChIP-qPCR assays only when cells were transfected with the PIK3CA E545K vector (Fig. 6f, bottom panel and Supplementary Fig. 23a). Thus, ELK1 transcriptional activity is enhanced by mutant PIK3CA in both head and neck and BRCA models. RT-qPCR analysis demonstrated an increase in the mRNA levels of four TCF4 target genes in Cal27 cells with PIK3CA E545K compared to WT Cal27 and control cells (Fig. 6g, top panel and Supplementary Fig. 23b). Increased occupancy of TCF4 at the promoters of these genes was confirmed by ChIP assays only when cells were transfected with the PIK3CA E545K vector (Fig. 6g, bottom panel and Supplementary Fig. 23b). Thus, mutant PIK3CA enhances TCF4 transcriptional activity in head and neck as well as BRCA models.

FOXO1 activity was not associated with mutant PIK3CA in our HNSC model. Indeed, RT-qPCR analysis demonstrated no significant change in the mRNA levels of the FOXO1 target genes in PIK3CA E545K Cal27 cells compared to WT Cal27 cells and control cells (Fig. 6h, top panel and Supplementary Fig. 23c). Further, no change in occupancy of FOXO1 at the promoters of TNFSF10 and RUNX1 was shown by ChIP assays when cells were transfected with the PIK3CA E545K vector (Fig. 6h, bottom panel and Supplementary Fig. 23c).

ELK1 is phosphorylated through activation of the MAPK/ERK pathways and translocates to the nucleus, resulting in activation/ repression of downstream targets ${ }^{47,49-52}$ that are important in cell proliferation, apoptosis, cell migration and invasion, and inflammatory response ${ }^{53,54}$. Immunohistochemistry in breast tumour specimens has shown that the levels of p-ELK1 expression are significantly elevated in luminal and Her-2-negative BRCA subtypes ${ }^{55}$, but how Elk1 is activated in BRCA is not known. Our computational and experimental results suggest that a potential mechanism for ELK1 activation is through an activating PIK3CA mutation.

TCF4 interacts with $\beta$-catenin to mediate Wnt signalling and has been implicated in colorectal tumorigenesis 56,57 . Our analyses confirm that TCF4 activation may result from an activating PIK3CA mutation. Recently, a small-molecule inhibitor of the $\beta$-catenin/TCF4 interaction called LF3 has been shown to diminish Wnt-dependent biologic characteristics of colon cancer cells, inhibit their self-renewal capacity and induce their differentiation ${ }^{58}$. Since we demonstrated altered transcriptional activity of TCF4 downstream of mutant PIK3CA in breast and head and neck cancer cells, targeting TCF4 might be new therapeutic strategy in PIK3CA mutant patients.

FOXO TFs, including FOXO1, are implicated in the regulation of stress resistance, metabolism, cell cycle, apoptosis and DNA repair. It is well known that constitutive PI3K-AKT pathway activation causes downregulation of FOXO tumour suppressor functions in $\mathrm{BRCA}^{59}$. However, regulation of FOXO target genes is multifactorial, and based on our findings, contextdependent. Specifically, we showed that an activating PIK3CA mutation altered FOXO1 activity in the BRCA model but not in the head and neck cancer model, consistent with the contextspecific predictions of our algorithm. This shows one example of how a clinically relevant 'actionable mutation' impacts regulatory programs in a cancer-specific manner, giving clues about druggability across tumour types.

\section{Discussion}

Many targetable alterations are present across multiple tumour types. For example, activating mutations and amplifications of PIK3CA are targetable by PI3K inhibitors, which are in active clinical assessment in combination therapies with RTK inhibitors and antioestrogen therapies in BRCA, antiandrogen therapy in PRAD and MEK inhibitors in many solid tumours ${ }^{60-65}$. However, the cancer-specific context can impact how patients respond to targeted therapies, since the targeted protein resides in a network of interacting proteins and is subject to extensive feedback and crosstalk between signalling pathways. In recent clinical trials of targeted therapies (for example, Gleevec in chronic myelogenous leukaemia, herceptin in BRCA, BRAF inhibitors in melanoma), patients who share the targeted mutation and tumour type displayed highly variable responses to the drugs ${ }^{66}$. Therefore, a systematic stratification of tumours that goes beyond therapeutically actionable alterations and incorporates other functional readouts-for example, dysregulated TF and (phospho)protein signatures derived from our model-may better predict which patients will benefit from targeted and combination therapies.

Using inferred TF/protein activities in tumours may also reveal clinically relevant patient subgroups. Patients with endometrioid carcinomas display heterogeneous clinical courses and response to therapy, despite similar tumour histopathology. Clustering UCEC tumours by TF activities revealed a subclass of endometrioid tumours that correlated with $\beta$-catenin mutation status and had poorer survival (Supplementary Fig. 24, $P<0.001$ ). Linking mutant $\beta$-catenin to putative downstream TF effectors could inform future mechanistic studies-for example, short hairpin RNA or CRISPR/Cas screening to identify TFs whose deletion/knockdown leads to changes in proliferation-to develop new therapeutic strategies.

Previous algorithms to interpret the role of somatic alterations have examined enrichment of mutations in known pathways ${ }^{3,4,6}$ or searched for alterations that represent mutual exclusive patterns $^{2}$, subnetworks ${ }^{10,67-69}$ or modules ${ }^{70}$. These approaches examine co-occurrences of somatic alterations in a known protein interaction network without explicitly modelling their impact on transcriptional programs or signalling. Several recent studies have integrated TF binding site or occupancy data to identify cancer-associated TFs, for example, combining tumourspecific DNA methylation changes in distal enhancers, mRNA sequencing and cis-regulatory sequences mediating effects on target genes ${ }^{71}$ or integrating ENCODE TF ChIP-seq profiles with the pancancer TCGA expression data ${ }^{72}$. However, these approaches do not model the relationship between perturbed pathways (for example, from proteomic data) and TF activity, nor do they consider the impact of somatic alterations on gene regulatory models.

Overall, current methods cannot translate the mutational landscape of a tumour into a usable model of affected pathways nor use mutational status to predict accurately response to targeted therapies. Our model is designed to capture the causal flow of information from signalling to TFs to target genes; the association analysis is likely to identify causal impacts of mutations and copy number events, since somatic alterations usually alter TF activity/signalling rather than vice versa 
(with exception of TFs/signalling pathways involved in DNA repair). Our analysis revealed both known and putative interactions of frequently altered genes with signalling and transcriptional programs in a pancancer context and provides a general strategy for future studies. In cases where a mutation is associated with the altered activity of a targetable $\mathrm{TF}$ or (phospho)protein, our analysis may suggest combination therapies.

The method we have presented has several limitations. First, our analysis uses predicted TF binding sites based on existing $\mathrm{TF}$ motif databases and restricted to promoter sequences; therefore, the TF motif hit matrix is noisy, incomplete and not context-specific. Indeed, due to the strong correlation structure between RPPA and mRNA expression data, AR models trained with the true motif hit matrix achieve only a modest-albeit significant-improvement in prediction performance over models trained on randomized motif data (Supplementary Table 1). Additionally, since many inferred TF and (phospho)protein activities are correlated, individual genomic aberrations may be associated with many regulators. This multiplicity of inferred effects may be biologically reasonable but complicates interpretation. Another methodological challenge is the need to control for the complex background of genomic aberrations. To do this, we used regularized regression with permutation testing to identify a smaller set of somatic alterations with confident associations. Still, the problem of selecting a few significant covariates from a long candidate list given limited sample size is inherently difficult with no fail-safe solution.

Despite these limitations, we have presented a principled integrative strategy for predicting the context-specific impact of somatic alterations on transcriptional programs and signalling pathways. Moreover, our predictions generalize to independent patient cohorts and validate experimentally in isogenic cancer cell line models. We anticipate that such integrative statistical modelling strategies will be crucial for personalizing cancer therapies.

\section{Methods}

Data and preprocessing. We downloaded RPPA protein expression data from TCPA (http://bioinformatics.mdanderson.org/main/TCPA:Overview). RPPA protein expression data for the UCS study, RNA-seq gene expression data, somatic mutation data and clinical data were downloaded from TCGA's Firehose data run (https://confluence.broadinstitute.org/display/GDAC/Dashboard-Stddata). GISTIC copy number data was downloaded from TCGA's Firehose analyses run (https://confluence.broadinstitute.org/display/GDAC/Dashboard-Analyses). Only the samples 'whitelisted' by TCGA for the Pan-Cancer Analysis Working Group were used in the study. For our analysis, we restricted to samples with parallel RNA-seq, RPPA, somatic mutation and GISTIC copy number data (Supplementary Table 14).

Silent mutations were filtered from somatic mutation data. We removed genes that were not identified as significant $(q<0.05)$ by the MutSigCV ${ }^{73}$ as well as not present at least ten samples in each cancer type. To determine copy number alteration events, we used the set of discrete copy number calls provided by GISTIC2 (ref. 74). We considered genes to be altered only in samples where they resided either in regions of homozygous loss $(-2)$ or high-level amplification (2) among the set of recurrent copy number alterations. We removed genes that were not identified as significant $(q<0.001)$ by the GISTIC2 (ref. 74) as well as not present at least ten samples in each cancer type. Then, we encoded somatic aberrations as being present/absent. The final selected set of binary calls for genomic alterations provided a simplified but informative description of the somatic alterations observed in individual tumours.

Log 10-transformed RNA-seq RSEM gene expression values for each of the 12 cancer types were processed independently to identify the set of 5,000 genes that varied most across samples. Gene expression and protein expression vectors were both mean-centred.

To construct the motif hit matrix, we downloaded the TF binding site predictions (TRANSFAC v.7.4) for all target genes from $\mathrm{MSigDB}^{34}$. We removed motifs with similar sets of targets to reduce redundancy. This matrix defined a candidate set of regulatory relationships between TFs and target genes. Further, for each of the 12 cancer types, we filtered TFs that were not expressed in at least $40 \%$ of samples (Supplementary Data 1).
We obtained SILAC-based quantitative phosphoproteomic data set of a spontaneously immortalized non-tumorigenic breast epithelial cell line MCF10A along with two isogenic derivatives generated by knock-in of mutant alleles-one bearing the E545K mutation and the other bearing the H1047R mutation of the PIK3CA gene-from the originally published Supplementary Data ${ }^{45}$. We used a 1.5 -fold cutoff value to designate peptides as having increased phosphorylation and a 0.67 -fold for decreased phosphorylation (same thresholds as original publication $)^{45}$. We also obtained human protein microarray-based AKT1 kinase assays from the originally published Supplementary Data ${ }^{45}$.

We obtained RPPA data for uterine corpus endometrioid carcinoma $a^{25,75}$ and head and neck cancer patients ${ }^{28}$ from the original publications.

Training the AR models. AR is an algorithm for efficiently solving a regularized bilinear regression problem ${ }^{15,16}$, defined here as follows. For a data set of $M$ tumour samples profiled using RNA-seq with $N$ genes, we let $\mathbf{Y} \in R^{N \times M}$ be the mean-centred $\log 10$ gene expression profiles of tumour samples. Each column of $\mathbf{Y}$ corresponds to an RNA-seq experiment. We define each gene's TF attributes in a matrix $\mathbf{D} \in R^{N \mathrm{NQ}}$, where each row represents a gene and each column represent the hit vector for a TF, that is, the bit vector indicating whether there is binding site for the TF in the promoter region of each gene. We define the RPPA attributes of tumour samples as a matrix $\mathbf{P} \in R^{M \times S}$ where each row represents a tumour sample and each column represents the (mean-centred) log RPPA protein expression profile for the tumour sample. We set up a bilinear regression problem to learn the weight matrix $\mathbf{W} \in R^{Q \times S}$ on paired of TF signalling protein features:

$$
\mathbf{D W P}^{\mathrm{T}}+\varepsilon=\mathbf{Y}
$$

We can transform the system to an equivalent system of equations by reformulating the matrix products as Kronecker products

$$
\mathbf{D W} \mathbf{P}^{\mathrm{T}} \Leftrightarrow(\mathbf{P} \otimes \mathbf{D}) \operatorname{vec}(\mathbf{W})
$$

where $\otimes$ is a Kronecker product and vec(.) is a vectorizing operator that stacks a matrix and produces a vector, yielding a standard (if large-scale) regression problem. Full details and a derivation of the reduced optimization problem are provided elsewhere ${ }^{16}$. We fit the ridge regression model using the SLEP MATLAB package and evaluate performance with 10 -fold cross-validation.

Given the (phospho)protein profile of a test tumour sample (centred relative to the mean of the training set), we can right multiply the (phospho)protein expression vector through the trained model to predict the similarity of its expression profile to those of the training tumour samples. To recover a reconstruction of the test gene expression profile from the predicted similarities, we assume that the test expression profile is in the linear span of the training profiles. Then, a simple transformation converts the vector of computed similarities into a predicted gene expression variation profiles ${ }^{16}$. Finally, to infer the (phospho)protein activity in a new sample from the (centred) gene expression profile, we can left multiply through the model via $\mathbf{Y}^{\mathrm{T}} \mathrm{DW}$ and to infer the TF activities in each sample, we can right-multiply the protein expression profiles through the model by $\mathbf{W P}^{\mathrm{T}}$.

Significance analysis for TF and (phospho)protein activities. To assess the statistical significance of the inferred (phospho)protein and TF activities obtained from the model via the $\mathbf{Y}^{\mathrm{T}} \mathbf{D W}$ and $\mathbf{W P}^{\mathrm{T}}$ mappings, respectively, we developed an empirical null model as follows. First, we generated random permutations of the gene expression profiles $\mathbf{Y}$ for each tumour type. For each permuted $\mathbf{Y}$ response matrix, we trained an AR model using true $\mathbf{D}$ and $\mathbf{P}$ input matrices and computed the corresponding inferred TF and (phospho)protein activities via the $\mathbf{Y}^{\mathrm{T}} \mathbf{D W}$ and $W P^{\mathrm{T}}$ mappings. Using this permutation and model fitting procedure 5000 times, we generated an empirical null model for TF and (phospho)protein activity distribution for each sample. To identify significant regulator activities (R), we assessed the nominal $P$ value for each sample relative to the empirical null model for the particular regulator ( $\mathrm{TF} /($ phospho)protein), and we corrected for multiple hypothesis testing of non-independent hypotheses using the Benjamini-HochbergYekutieli procedure. Then, we reported the significant regulators using an FDR of 0.1 for the largest TCGA studies (BRCA, KIRC; $>300$ samples), an FDR of 0.15 for mid-size studies (BLCA, COADREAD, HNSC, LUAD, LUSC, UCEC, OV, PRAD; sample size $<300$ and $>100$ ), and an FDR of 0.25 for small studies (GBM, UCS $<100$ samples) as our thresholds for significance. Then, we calculated, for each $\mathrm{TF} /$ signalling regulator, the frequency over samples where the regulator passed its significant threshold for a given cancer. We used this approach to identify significant regulators in each cancer type to identify the shared and cancer-specific roles $\mathrm{TF} /($ phospho)protein regulators.

Model impact of genomic aberrations in terms of TF/(phospho)protein activity We used ridge regression to predict each $\mathrm{TF} /$ (phospho)protein regulator's activity (R) from genomic aberration profiles and used a permutation test approach to assign significance to ridge regression coefficients. Somatic alterations were simply encoded as present/absent. In the permutation test, the elements of the outcome vector of regulator activities $\mathbf{R}$ were randomly permuted across samples, and the ridge regression model was fitted using the permuted observations to obtain ridge regression coefficients. By performing 10,000 such permutations, a null distribution of the regression coefficients was generated. The permutation test $P$ value was 
calculated as the proportion of regression coefficients from the null distribution greater than or equal in absolute value to the absolute value of the coefficient fitted to the true (non-permuted) data. Further we corrected for multiple hypothesis testing of non-independent hypotheses using the Benjamini-Hochberg-Yekutieli procedure across all $\mathrm{TF} /$ signalling regulators for each cancer, and we multiplied these FDR-adjusted $P$ values with the sign of the coefficient from the model to calculate a final regulatory-genomic aberration association score. For downstream analysis, we restricted our analysis to regulators identified as significant in at least $1 \%$ of samples in each cancer and genomic aberrations with FDR-corrected $P<0.15$ across regulators from the ridge regression analysis with permutation test. We also assessed synergistic/antagonistic effects of pairs of genomic aberrations on TF/protein activity by building linear models with interaction terms for each pair of genomic aberrations. We restricted our analysis to pairs of genes that were altered in at least in 20 tumour samples as well as comutated in at least 10 tumour samples. Here the activity of each $\mathrm{TF} /$ (phospho)protein regulator (R) was a modelled function of somatic aberration pairs $A$ and $B$ :

$$
R_{i j k}=\mu+A_{i}+B_{j}+(A B)_{i j}+\varepsilon_{k}
$$

where $A_{i}$ and $B_{j}$ represent the main effects of the $i$ th and $j$ th values of $A$ and $B$, respectively, $(A B)_{i j}$ is the effect of the interaction in that combination, and $\varepsilon_{k}$ is the error term of the $k$ th observation in that combination. Here the genomic aberrations are binarized (present/absent), so the values are 1 and 0 .

We first looked for regulator models for which the coefficient of the interaction term was significant $(P<0.05)$. If the interaction term was significant, and if its coefficient was greater than zero and greater than the coefficients of the genomic aberration pair, we assumed this pair of somatic aberrations had a synergistic effect on regulator activity. If the interaction term was significant and its coefficient was less than zero and less than the coefficients of the genomic aberration pair, we assumed this pair of somatic aberrations had an antagonistic effect on regulator activity.

Survival analyses. We built Cox proportional hazard regression models for regulators that attained significance by our empirical $P$ value procedure in at least $5 \%$ of samples using (1) their inferred TF activity profiles and (2) their gene expression profiles corresponding to TFs. We used clinical stage as a background factor for BLCA and KIRC and histological subtype as a background factor for UCEC. Overall survival was calculated from the date of initial diagnosis of cancerto disease-specific death (patients whose vital status is termed dead) and months to last follow-up (for patients who are alive). Further, we evaluated prognostic accuracy of survival models using a log-rank test. We corrected for multiple hypothesis testing of non-independent hypotheses using the FDR procedure across all models in each cancer type. FDR-corrected $P$ values of models built from inferred TF activities and actual TF mRNA expression profiles were compared using one-sided paired Wilcoxon's signed-rank test.

For the validation set, we used the TCGA-trained UCEC model to infer TF activity profiles of MDACC $(n=178)$ and Bergen $(n=209)$ data sets $^{75}$ from their RPPA profiles. Since these data sets do not include serous endometrial carcinoma samples, we build univariate Cox models with just TCGA endometrioid endometrial carcinoma patients. We first identified TFs with univariate Cox $P<0.05$ on the TCGA patients. Then, we predicted the risk for each patient in the validation set and calculating concordance index. We reported the $P$ value for the statistical test if the concordance index estimate was different from 0.5 .

For visualization, Kaplan-Meier survival analysis was used to show the association of the inferred TF activity with patient survival. For each selected TF and cancer-type combination, each patient's risk was calculated, and patients were ranked in descending order. We designated the top $40 \%$ of the patients as the high-risk group and the bottom $40 \%$ as the low-risk group.

Statistical analysis. Statistical tests were performed with the R statistical environment. For population comparisons of inferred TF and (phospho)protein activities, we performed two-tailed Wilcoxon's signed-rank tests and determined the direction of shifts by comparing the mean of two populations.

Cell lines and transfection. The BRCA cell line, MCF7, which has a PIK3CA E545K mutation, and the targeted correction of the E545K mutation to WT PIK3CA were obtained from the Lauring $\mathrm{Lab}^{46}$. The head and neck cancer cell line, Cal27, was obtained from the American Type Culture Collection. The cell lines have been tested negative for mycoplasma contamination. Parental and WT PIK3CA MCF7 and Cal27 were maintained in Dulbecco's modified Eagle's medium (DMEM/DF12) supplemented with $5 \%$ foetal bovine serum and $100 \mathrm{U} \mathrm{ml}^{-1}$ penicillin and $100 \mu \mathrm{g} \mathrm{ml}^{-1}$ streptomycin. Cal27 cell lines were transfected with pbabe control vector, pbabe WT PIK3CA and pbabe E545K PIK3CA vectors (Addgene) using Lipofectamine 3000 according to the manufacturer's instructions.

RNA extraction and quantitative real-time PCR. Total RNA was extracted from MCF7 and Cal27 cell lines using an RNA Extraction Kit from Qiagen. cDNA synthesis was performed using iScript from Bio-Rad, according to the manufacturer's instructions. The Applied Biosystems SYBR green mix (Life Technologies) was used to amplify specific genes listed in Supplementary Table 15

Primers used for mRNA expression were: ACTR3, 5' -CATTCCTG TGGCTGAAGGGT- $3^{\prime}$ and $5^{\prime}$-ATCGCTGCATGTGGTGTGTA- $3^{\prime}$; FOXP4, $5^{\prime}$-GACCCTGTGTGAAGACCTGG- ${ }^{\prime}$ and $5^{\prime}$-GTCAGGGGTTTCCAGGATG G-3 ${ }^{\prime} ;$ DDX27, $5^{\prime}$-TTGGGGAAGGACATCTGTGC- $3^{\prime}$ and $5^{\prime}$-CGGATCCGGA TGAACTCCTG- $3^{\prime}$ PAPLN, $5^{\prime}$-AGGTCATCTGTGCCATTGGG- $3^{\prime}$ and 5'-TGTAGAAGCCACTGCCCTTG-3'; PSMB4, 5' -GACATGCTGGGATCCTA CGG- $3^{\prime}$ and $5^{\prime}$-CTTTTTCGGTGACAGTGGCG-3'; WNK1, $5^{\prime}$-CTTTTT CGGTGACAGTGGCG- $3^{\prime}$ and $5^{\prime}$-CTTGGCTGTTCACTGTTGCC- $3^{\prime}$; CDK1, $5^{\prime}$-ACAGGTCAAGTGGTAGCCATG- $3^{\prime}$ and $5^{\prime}$-GGAGTGCCCAAAGCTC TGAA-3'; CAMKK1, $5^{\prime}$-CAGGAAGCTATCTGGAGGCG- ${ }^{\prime}$ and $5^{\prime}$-AAGTA CTCGAGGCCCAGGAT-3'; TNFSF10, 5-CCTCAGAGAGTAGCAGCTCACA-3' and $5^{\prime}$-CAGAGCCTTTTCATTCTTGGA- ${ }^{\prime} ;$ ACTB, $5^{\prime}$-CGTCTTCCCCTCCAT CGT $-3^{\prime}$ and $5^{\prime}$-GAAGGTGTGGTGCCAGATTT- $3^{\prime}$; APC, $5^{\prime}$-CATTTCCAAGA AGAGGGTTTGT- $3^{\prime}$ and $5^{\prime}$-GATCAGCAAGAAGCAATGACC- $3^{\prime}$; FBXW11, $5^{\prime}$-GGCTGCCGTCAATGTAGTAGA- $3^{\prime}$ and $5^{\prime}$-GTGCTCGTGCTCCAG ACTT- $3^{\prime}$; PPP2R5E, 5'-GTGTGTATCTAGCCCCCATTTT- $3^{\prime}$ and $5^{\prime}$-AAACTCA TGATGTATTCATTATTCCAA- $3^{\prime}$; WNT10B, $5^{\prime}$-ATGCGAATCCACAAC AACAG- $3^{\prime}$ and $5^{\prime}$-TCCAGCATGTCTTGAACTGG-3'.

Chromatin immunoprecipitation. MCF7 and Cal27 cell lines were crosslinked with $1 \%$ formaldehyde for $10 \mathrm{~min}$ at room temperature and quenched with $125 \mathrm{mM}$ glycine for $5 \mathrm{~min}$ at room temperature. Cell were lysed and sheared to obtain chromatin fragments of 200-500 bp. Sheared chromatin was incubated overnight with $2 \mu \mathrm{g}$ of rabbit monoclonal ChIP grade antibody to ELK1 (E277, ab32106; Abcam) as has been previously used by Zhang et al. ${ }^{76}$ $2 \mu \mathrm{g}$ of a goat polyclonal antibody to TCF4 (N-20, sc-8631; Santa Cruz) as has been previously used by Ding et al. ${ }^{77}$ and $2 \mu \mathrm{g}$ of rabbit polyclonal FOXO1 (H-108, sc-11350; Santa Cruz) as has been previously used by Xiong et al. ${ }^{78}$ Protein G magnetic beads were used to capture antibody-chromatin association overnight, followed by sequential washes. The antibody bound beads were then reverse crosslinked for $6 \mathrm{~h}$ at $65^{\circ} \mathrm{C}$, followed by proteinase $\mathrm{K}$ treatment at $55^{\circ} \mathrm{C}$ for $1 \mathrm{~h}$. The ChIP DNA was purified using a DNA Purification Kit from Qiagen. The Applied Biosystems SYBR green mix was used to amplify specific regions (Supplementary Table 16).

Western blot analysis. Cells were lysed and proteins were extracted in RIPA buffer that was supplemented with phosphatase and protease inhibitors. Proteins were separated by SDS-polyacrylamide gel electrophoresis gels and transferred to a PVDF (polyvinylidene difluoride) membrane. Membranes with blocked with $5 \%$ bovine serum albumin and probed using specific antibodies. Actin $(1: 2,000)$, pAKT (S473) (1:1,000), pS6K (T389) (1:1,000), HA (1:1,000) were all from Cell Signaling Technology (CST).

Data availability. RPPA protein expression data is available in a public repository from TCPA (http://bioinformatics.mdanderson.org/main/TCPA:Overview). RPPA protein expression data for the UCS study, RNA-seq gene expression data, somatic mutation data and clinical data are available in a public repository from TCGA's Firehose data run (https://confluence.broadinstitute.org/display/GDAC/Dashboard-Stddata). GISTIC copy number data is available in a public repository from TCGA's Firehose analyses run (https://confluence.broadinstitute.org/display/ GDAC/Dashboard-Analyses). Only the samples 'whitelisted' by TCGA for the Pan-Cancer Analysis Working Group were used in the study. For our analysis, we restricted to samples with parallel RNA-seq, RPPA, somatic mutation and GISTIC copy number data (Supplementary Table 14 and Supplementary Data 1).

The authors declare that all data supporting the findings of this study are available within the article and its Supplementary Information files or from the corresponding author on reasonable request.

\section{References}

1. Hanahan, D. \& Weinberg, R. A. The hallmarks of cancer. Cell 100, 57-70 (2000).

2. Ciriello, G., Cerami, E., Sander, C. \& Schultz, N. Mutual exclusivity analysis identifies oncogenic network modules. Genome Res. 22, 398-406 (2012).

3. Cancer Genome Atlas Research, N. Comprehensive genomic characterization defines human glioblastoma genes and core pathways. Nature 455, 1061-1068 (2008).

4. Cancer Genome Atlas, N. Comprehensive molecular characterization of human colon and rectal cancer. Nature 487, 330-337 (2012).

5. Cancer Genome Atlas, N. Comprehensive genomic characterization of head and neck squamous cell carcinomas. Nature 517, 576-582 (2015).

6. Cancer Genome Atlas Research, N. Integrated genomic analyses of ovarian carcinoma. Nature 474, 609-615 (2011).

7. Cancer Genome Atlas Research, N. Comprehensive genomic characterization of squamous cell lung cancers. Nature 489, 519-525 (2012). 
8. Cancer Genome Atlas Research, N. Comprehensive molecular characterization of clear cell renal cell carcinoma. Nature 499, 43-49 (2013).

9. Cancer Genome Atlas Research, N. et al. Integrated genomic characterization of endometrial carcinoma. Nature 497, 67-73 (2013).

10. Cerami, E., Demir, E., Schultz, N., Taylor, B. S. \& Sander, C. Automated network analysis identifies core pathways in glioblastoma. PLOS ONE 5, e8918 (2010).

11. Hofree, M., Shen, J. P., Carter, H., Gross, A. \& Ideker, T. Networkbased stratification of tumor mutations. Nat. Methods 10, 1108-1115 (2013).

12. Costello, J. C. et al. A community effort to assess and improve drug sensitivity prediction algorithms. Nat. Biotechnol. 32, 1202-1212 (2014).

13. Hyman, D. M. et al. Vemurafenib in multiple nonmelanoma cancers with BRAF V600 mutations. N. Engl. J. Med. 373, 726-736 (2015).

14. Juric, D. et al. in Proc. 103rd Meeting of AACR CT-01 (2012).

15. Osmanbeyoglu, H. U., Pelossof, R., Bromberg, J. F. \& Leslie, C. S. Linking signaling pathways to transcriptional programs in breast cancer. Genome Res. 24, 1869-1880 (2014).

16. Pelossof, R. et al. Affinity regression predicts the recognition code of nucleic acid-binding proteins. Nat. Biotechnol. 33, 1242-1249 (2015).

17. Catalano, S. et al. Evidence that leptin through STAT and CREB signaling enhances cyclin D1 expression and promotes human endometrial cancer proliferation. J. Cell Physiol. 218, 490-500 (2009).

18. Ishida, M. et al. Activation of aryl hydrocarbon receptor promotes invasion of clear cell renal cell carcinoma and is associated with poor prognosis and cigarette smoke. Int. J. Cancer 137, 299-310 (2015).

19. Takaha, N. et al. Expression and role of HMGA1 in renal cell carcinoma. J. Urol. 187, 2215-2222 (2012).

20. Park, J. T., Shih, Ie, M. \& Wang, T. L. Identification of Pbx1, a potential oncogene, as a Notch 3 target gene in ovarian cancer. Cancer Res. 68, 8852-8860 (2008).

21. Kikugawa, T. et al. PLZF regulates $\mathrm{Pbx} 1$ transcription and $\mathrm{Pbx} 1-\mathrm{HoxC} 8$ complex leads to androgen-independent prostate cancer proliferation. Prostate 66, 1092-1099 (2006).

22. Magnani, L., Ballantyne, E. B., Zhang, X. \& Lupien, M. PBX1 genomic pioneer function drives ERalpha signaling underlying progression in breast cancer. PLoS Genet. 7, e1002368 (2011).

23. Magnani, L. et al. The pioneer factor PBX1 is a novel driver of metastatic progression in ERalpha-positive breast cancer. Oncotarget 6, 21878-21891 (2015).

24. Kim, T. H. et al. Forkhead box O-class 1 and forkhead box G1 as prognostic markers for bladder cancer. J. Korean Med. Sci. 24, 468-473 (2009)

25. Liang, H. et al. Whole-exome sequencing combined with functional genomics reveals novel candidate driver cancer genes in endometrial cancer. Genome Res. 22, 2120-2129 (2012).

26. Chen, J. Signaling pathways in HPV-associated cancers and therapeutic implications. Rev. Med. Virol. 25(suppl 1): 24-53 (2015).

27. Hu, Z. et al. Genome-wide profiling of HPV integration in cervical cancer identifies clustered genomic hot spots and a potential microhomologymediated integration mechanism. Nat. Genet. 47, 158-163 (2015).

28. Sewell, A. et al. Reverse-phase protein array profiling of oropharyngeal cancer and significance of PIK3CA mutations in HPV-associated head and neck cancer. Clin. Cancer Res. 20, 2300-2311 (2014).

29. Westra, W. H. et al. Inverse relationship between human papillomavirus-16 infection and disruptive p53 gene mutations in squamous cell carcinoma of the head and neck. Clin. Cancer Res. 14, 366-369 (2008).

30. Scheffner, M., Takahashi, T., Huibregtse, J. M., Minna, J. D. \& Howley, P. M. Interaction of the human papillomavirus type $16 \mathrm{E} 6$ oncoprotein with wild-type and mutant human p53 proteins. J. Virol. 66, 5100-5105 (1992).

31. Carro, M. S. et al. The transcriptional network for mesenchymal transformation of brain tumours. Nature 463, 318-325 (2010).

32. Ignatiadis, M. \& Sotiriou, C. Luminal breast cancer: from biology to treatment. Nat. Rev. Clin. Oncol. 10, 494-506 (2013).

33. Reis-Filho, J. S. et al. Is TTF1 a good immunohistochemical marker to distinguish primary from metastatic lung adenocarcinomas? Pathol. Res. Pract. 196, 835-840 (2000).

34. Liu, Y. et al. Clinical significance of CTNNB1 mutation and Wnt pathway activation in endometrioid endometrial carcinoma. J. Natl Cancer Inst. 106, dju245 (2014)

35. Cowey, C. L. \& Rathmell, W. K. VHL gene mutations in renal cell carcinoma: role as a biomarker of disease outcome and drug efficacy. Curr. Oncol. Rep. 11, 94-101 (2009).

36. Leinonen, H. M., Kansanen, E., Polonen, P., Heinaniemi, M. \& Levonen, A. L. Role of the Keap1-Nrf2 pathway in cancer. Adv. Cancer Res. 122, 281-320 (2014).

37. Cantley, L. C. The phosphoinositide 3-kinase pathway. Science 296, 1655-1657 (2002).
38. Engelman, J. A. Targeting PI3K signalling in cancer: opportunities, challenges and limitations. Nat. Rev. Cancer 9, 550-562 (2009).

39. Janku, F. et al. Assessing PIK3CA and PTEN in early-phase trials with PI3K/AKT/mTOR inhibitors. Cell Rep. 6, 377-387 (2014).

40. Joshi, A., Miller, Jr. C., Baker, S. J. \& Ellenson, L. H. Activated mutant p110alpha causes endometrial carcinoma in the setting of biallelic Pten deletion. Am. J. Pathol. 185, 1104-1113 (2015).

41. Britschgi, A. et al. JAK2/STAT5 inhibition circumvents resistance to $\mathrm{PI} 3 \mathrm{~K} / \mathrm{mTOR}$ blockade: a rationale for cotargeting these pathways in metastatic breast cancer. Cancer Cell 22, 796-811 (2012).

42. Cohen-Solal, K. A., Boregowda, R. K. \& Lasfar, A. RUNX2 and the PI3K/AKT axis reciprocal activation as a driving force for tumor progression. Mol. Cancer 14, 137 (2015).

43. Cabodi, S. et al. Convergence of integrins and EGF receptor signaling via PI3K/Akt/FoxO pathway in early gene Egr-1 expression. J. Cell. Physiol. 218, 294-303 (2009).

44. Ibrahim, Y. H. et al. PI3K inhibition impairs BRCA1/2 expression and sensitizes BRCA-proficient triple-negative breast cancer to PARP inhibition. Cancer Discov. 2, 1036-1047 (2012).

45. Wu, X. et al. Activation of diverse signalling pathways by oncogenic PIK3CA mutations. Nat. Commun. 5, 4961 (2014).

46. Beaver, J. A. et al. PIK3CA and AKT1 mutations have distinct effects on sensitivity to targeted pathway inhibitors in an isogenic luminal breast cancer model system. Clin. Cancer Res. 19, 5413-5422 (2013).

47. Odrowaz, Z. \& Sharrocks, A. D. ELK1 uses different DNA binding modes to regulate functionally distinct classes of target genes. PLoS Genet. 8, e1002694 (2012).

48. Vasquez, Y. M. et al. FOXO1 is required for binding of PR on IRF4, novel transcriptional regulator of endometrial stromal decidualization. Mol. Endocrinol. 29, 421-433 (2015).

49. Boros, J. et al. Elucidation of the ELK1 target gene network reveals a role in the coordinate regulation of core components of the gene regulation machinery. Genome Res. 19, 1963-1973 (2009).

50. Gille, H. et al. ERK phosphorylation potentiates Elk-1-mediated ternary complex formation and transactivation. EMBO J. 14, 951-962 (1995).

51. Odrowaz, Z. \& Sharrocks, A. D. The ETS transcription factors ELK1 and GABPA regulate different gene networks to control MCF10A breast epithelial cell migration. PLoS ONE 7, e49892 (2012).

52. Booy, E. P., Henson, E. S. \& Gibson, S. B. Epidermal growth factor regulates Mcl-1 expression through the MAPK-Elk-1 signalling pathway contributing to cell survival in breast cancer. Oncogene 30, 2367-2378 (2011).

53. Hipskind, R. A., Rao, V. N., Mueller, C. G., Reddy, E. S. \& Nordheim, A Ets-related protein Elk-1 is homologous to the c-fos regulatory factor p62TCF. Nature 354, 531-534 (1991).

54. Wyrzykowska, P., Stalinska, K., Wawro, M., Kochan, J. \& Kasza, A. Epiderma growth factor regulates PAI-1 expression via activation of the transcription factor Elk-1. Biochim. Biophys. Acta 1799, 616-621 (2010).

55. Laliotis, A. et al. Immunohistochemical study of pElk-1 expression in human breast cancer: association with breast cancer biologic profile and clinicopathologic features. Breast 22, 89-95 (2013).

56. Zhang, T. et al. Evidence that APC regulates survivin expression: a possible mechanism contributing to the stem cell origin of colon cancer. Cancer Res. 61, 8664-8667 (2001).

57. Schepeler, T. et al. Attenuation of the beta-catenin/TCF4 complex in colorectal cancer cells induces several growth-suppressive microRNAs that target cancer promoting genes. Oncogene 31, 2750-2760 (2012).

58. Fang, L. et al. A small-molecule antagonist of the beta-catenin/TCF4 interaction blocks the self-renewal of cancer stem cells and suppresses tumorigenesis. Cancer Res. 76, 891-901 (2016).

59. Bullock, M. FOXO factors and breast cancer: outfoxing endocrine resistance. Endocr. Relat. Cancer 23, R113-R130 (2016).

60. Baselga, J. et al. Everolimus in postmenopausal hormone-receptor-positive advanced breast cancer. N. Engl. J. Med. 366, 520-529 (2012).

61. Beaver, J. A. \& Park, B. H. The BOLERO-2 trial: the addition of everolimus to exemestane in the treatment of postmenopausal hormone receptor-positive advanced breast cancer. Fut. Oncol. 8, 651-657 (2012).

62. Ma, C. X. et al. A phase 1 trial of BKM120 (Buparlisib) in combination with fulvestrant in postmenopausal women with estrogen receptor positive metastatic breast cancer.. Clin. Cancer Res. 22, 1583-1591 (2015).

63. Bedard, P. L. et al. A phase Ib dose-escalation study of the oral pan-PI3K inhibitor buparlisib (BKM120) in combination with the oral MEK1/2 inhibitor trametinib (GSK1120212) in patients with selected advanced solid tumors. Clin. Cancer Res. 21, 730-738 (2015).

64. Shah, P. D. et al. Phase I trial of daily PI3K $\alpha$ inhibitor BYL719 plus letrozole (L) or exemestane (E) for patients (pts) with hormone receptor-positive $(\mathrm{HR}+$ ) metastatic breast cancer (MBC).. J. Clin. Oncol. 32, 5s (2014). 
65. Rodon, J., Dienstmann, R., Serra, V. \& Tabernero, J. Development of PI3K inhibitors: lessons learned from early clinical trials. Nat. Rev. Clin. Oncol. 10, 143-153 (2013).

66. Sharma, S. V., Haber, D. A. \& Settleman, J. Cell line-based platforms to evaluate the therapeutic efficacy of candidate anticancer agents. Nat. Rev. Cancer 10, 241-253 (2010)

67. Vandin, F., Upfal, E. \& Raphael, B. J. Algorithms for detecting significantly mutated pathways in cancer. J. Comput. Biol. 18, 507-522 (2011).

68. Vaske, C. J. et al. Inference of patient-specific pathway activities from multi-dimensional cancer genomics data using PARADIGM. Bioinformatics 26, i237-i245 (2010).

69. Chen, J. C. et al. Identification of causal genetic drivers of human disease through systems-level analysis of regulatory networks. Cell 159, 402-414 (2014).

70. Akavia, U. D. et al. An integrated approach to uncover drivers of cancer. Cell 143, 1005-1017 (2010).

71. Yao, L., Shen, H., Laird, P. W., Farnham, P. J. \& Berman, B. P. Inferring regulatory element landscapes and transcription factor networks from cancer methylomes. Genome Biol. 16, 105 (2015).

72. Jiang, P., Freedman, M. L., Liu, J. S. \& Liu, X. S. Inference of transcriptional regulation in cancers. Proc. Natl Acad. Sci. USA 112, 7731-7736 (2015).

73. Lawrence, M. S. et al. Mutational heterogeneity in cancer and the search for new cancer-associated genes. Nature 499, 214-218 (2013).

74. Mermel, C. H. et al. GISTIC2.0 facilitates sensitive and confident localization of the targets of focal somatic copy-number alteration in human cancers. Genome Biol. 12, R41 (2011).

75. Yang, J. Y. et al. Integrative protein-based prognostic model for earlystage endometrioid endometrial cancer. Clin. Cancer Res. 22, 513-523 (2016).

76. Zhang, L., Yang, S. H. \& Sharrocks, A. D. Rev7/MAD2B links c-Jun N-terminal protein kinase pathway signaling to activation of the transcription factor Elk-1. Mol. Cell. Biol. 27, 2861-2869 (2007).

77. Ding, Z. Y. et al. Smad6 suppresses the growth and self-renewal of hepatic progenitor cells. J. Cell Physiol. 229, 651-660 (2014).

78. Xiong, S., Salazar, G., Patrushev, N. \& Alexander, R. W. FoxO1 mediates an autofeedback loop regulating SIRT1 expression. J. Biol. Chem. 286, 5289-5299 (2011).

79. Bindea, G. et al. ClueGO: a Cytoscape plug-in to decipher functionally grouped gene ontology and pathway annotation networks. Bioinformatics 25, 1091-1093 (2009).

\section{Acknowledgements}

We thank Jacqueline Bromberg, Douglas Levine, Chris Sander, Christopher E. Barbieri, Petar Jelinic and James Hsieh for helpful discussions. The results published here are in whole or part based on data generated by The Cancer Genome Atlas pilot project established by the NCI and NHGRI (accession number: phs000178.v7p6). Information about TCGA and the investigators and institutions that constitute the TCGA research network can be found at http://cancergenome.nih.gov/. This work was supported by an award from NCI R21 CA205819. H.U.O. is supported by NCI award K99 CA207871. E.T. holds a fellowship from the Terri Brodeur Breast Cancer Foundation.

\section{Author contributions}

H.U.O. performed all computational experiments and analyses, helped to develop the algorithmic approaches and helped to write the paper. E.T. and C.C. performed the experimental validation and helped to write the experimental validation section. J.B. supervised the experimental validation. C.S.L. supervised the project, helped to develop the algorithmic approaches and helped to write the paper.

\section{Additional information}

Supplementary Information accompanies this paper at http://www.nature.com/ naturecommunications

Competing financial interests: The authors declare no competing financial interests.

Reprints and permission information is available online at http://npg.nature.com/ reprintsandpermissions/

How to cite this article: Osmanbeyoglu, H. U et al. Pancancer modeling predicts the context-specific impact of somatic mutations on transcriptional programs. Nat. Commun. 8, 14249 doi: 10.1038/ncomms14249 (2017).

Publisher's note: Springer Nature remains neutral with regard to jurisdictional claims in published maps and institutional affiliations.

(c) (i) This work is licensed under a Creative Commons Attribution 4.0 cc International License. The images or other third party material in this article are included in the article's Creative Commons license, unless indicated otherwise in the credit line; if the material is not included under the Creative Commons license, users will need to obtain permission from the license holder to reproduce the material. To view a copy of this license, visit http://creativecommons.org/licenses/by/4.0/

(C) The Author(s) 2017 